\title{
Free groups in lattices
}

\author{
LEWIS BOWEN
}

Let $G$ be any locally compact unimodular metrizable group. The main result of this paper, roughly stated, is that if $F<G$ is any finitely generated free group and $\Gamma<G$ any lattice, then up to a small perturbation and passing to a finite index subgroup, $F$ is a subgroup of $\Gamma$. If $G / \Gamma$ is noncompact then we require additional hypotheses that include $G=\operatorname{SO}(n, 1)$.

20E07; 20F65, 20F67, 22D40, 20E05

\section{Introduction}

Consider the following general problem. Let $\Gamma$ be a discrete cocompact subgroup of a locally compact metrizable unimodular group $G$. Can we use information about the subgroups of $G$ to infer the existence of subgroups of $\Gamma$ satisfying prescribed properties?

For example, suppose $G=\mathrm{PSL}_{2}(\mathbb{C})$, the group of orientation-preserving isometries of hyperbolic 3-space. $G$ contains a large variety of surface subgroups, that is, subgroups that are each isomorphic to the fundamental group of a closed surface of genus at least 2. It is a well-known open problem whether $\Gamma$, an arbitrary discrete cocompact subgroup of $G$, contains a surface subgroup.

This paper investigates the following strategy: given a subgroup $F<G$, attempt to change $F$ in some small way so that the resulting subgroup $F^{\prime}$ lies in $\Gamma$ and retains important properties of $F$. For example, we would like $F^{\prime}$ to be isomorphic to a finite-index subgroup of $F$ and the embedding $F^{\prime}<G$ to have asymptotic geometric properties close to those of $F$.

To be precise, let $F$ be an abstract group and $\phi: F \rightarrow G$ a homomorphism. Let $S \subset F$ be a finite symmetric generating set. Let $d$ be a left-invariant proper metric on $G$ inducing its topology. For $\epsilon>0$, we say that a map $\phi_{\epsilon}: F \rightarrow G$ is an $\epsilon$-perturbation of $F$ if

$$
d\left(\phi_{\epsilon}(f s), \phi_{\epsilon}(f) \phi(s)\right) \leq \epsilon
$$

for all $f \in F$ and $s \in S$. The map $\phi_{\epsilon}$ need not be a homomorphism. Indeed, we do not even require that it maps the identity element to the identity element. 
For example, if $G=\mathbb{R}, F=\mathbb{Z}$ and $\phi: \mathbb{Z} \rightarrow \mathbb{R}$ is the homomorphism $\phi(n)=n \tau$ for some number $\tau>0$ then $\phi_{\epsilon}: \mathbb{Z} \rightarrow \mathbb{R}$ need only satisfy $\left|\phi_{\epsilon}(n+1)-\phi_{\epsilon}(n)-\tau\right| \leq \epsilon$ for all $n \in \mathbb{Z}$.

We say that $\phi_{\epsilon}: F \rightarrow G$ is virtually a homomorphism if there exists a finite index subgroup $F^{\prime}<F$ such that

$$
\phi_{\epsilon}\left(f_{1} f_{2}\right)=\phi_{\epsilon}\left(f_{1}\right) \phi_{\epsilon}\left(f_{2}\right) \quad \forall f_{1} \in F^{\prime}, f_{2} \in F .
$$

If, in addition, $\phi_{\epsilon}\left(F^{\prime}\right)<\Gamma$ then we say it is virtually a homomorphism into $\Gamma$.

Theorem 1.1 (Main theorem: Uniform case) Let $G, \Gamma, d, F, S$ and $\phi$ be as above. Suppose $F$ is free and $S$ is a symmetric free generating set for $F$. Then for every $\epsilon>0$ there exists an $\epsilon$-perturbation $\phi_{\epsilon}$ of $\phi$ that is virtually a homomorphism into $\Gamma$.

I do not know if the theorem remains true if $F$ is required to be a surface group instead.

Theorem 1.2 (Main theorem: Nonuniform case) Let $G=\mathrm{SO}(n, 1), \Gamma<G$ be a discrete group with finite covolume, $F<G$ a convex cocompact free group, $S$ a symmetric free generating set for $F$ and $\epsilon>0$. Then there exists an $\epsilon$-perturbation $\phi_{\epsilon}$ of the inclusion map $\phi: F \rightarrow G$ that is virtually a homomorphism into $\Gamma$.

\subsection{Asymptotic Geometry}

Next we make a precise claim to the effect that, under special negative-curvature conditions, asymptotic geometric properties do not change much under an $\epsilon$-perturbation. The terms used below are standard. For the reader's convenience, they are listed in Section 6.1.

Let $(X, d)$ be a proper Gromov-hyperbolic space. Let $\partial X$ denote the Gromov boundary of $X$. Given a subset $Y \subset X \cup \partial X$, let $L(Y)=\partial X \cap \bar{Y}$ where $\bar{Y}$ denotes the closure of $Y$ in $X \cup \partial X$. If $\phi_{\epsilon}: H \rightarrow \operatorname{Isom}(X)$ is a map (where $H$ is an abstract group), then define the limit set of $\phi_{\epsilon}$ by $L\left(\phi_{\epsilon}\right)=L\left(\phi_{\epsilon}(H) p\right)$ where $p \in X$ is any point. $L\left(\phi_{\epsilon}\right)$ does not depend on the choice of $p$.

Fix a visual metric $d_{\partial}$ on $X$ with respect to some point $p \in X$. Let $d_{\text {Haus }}$ denote the Hausdorff distance on closed subsets of $\partial X$ with respect to $d_{\partial}$. Let $\mathcal{H D}$ denote the Hausdorff dimension of subsets of $\partial X$ with respect to $d_{\partial}$.

Theorem 1.3 Let $H$ be an abstract group with finite symmetric generating set $S$ and $\phi: H \rightarrow \operatorname{Isom}(X)$ be an injective homomorphism onto a quasi-convex cocompact subgroup of the group of isometries of $X$. Let $d_{\operatorname{Isom}(X)}$ be a left-invariant metric on 
Isom $(X)$ inducing the topology of uniform convergence on compact sets. Then for all $C>0$, there exists an $\epsilon_{0}>0$ such that if $0 \leq \epsilon \leq \epsilon_{0}$ and $\phi_{\epsilon}: H \rightarrow \operatorname{Isom}(X)$ is an $\epsilon$-perturbation of $\phi$ then

(1) $\phi_{\epsilon}$ is injective,

(2) if $\phi_{\epsilon}$ maps the identity in $H$ to the identity then $d_{\text {Haus }}\left(L\left(\phi_{\epsilon}\right), L(\phi)\right) \leq C$ and

(3) if $\phi_{\epsilon}$ is virtually a homomorphism then $\left|\mathcal{H D}\left(L\left(\phi_{\epsilon}\right)\right)-\mathcal{H D}(L(\phi))\right| \leq C$.

\subsection{Applications}

Let $\mathbb{H}^{n}$ denote $n$-dimensional hyperbolic space. Isom ${ }^{+}\left(\mathbb{H}^{n}\right)$, the group of orientationpreserving isometries of $\mathbb{H}^{n}$ is identified with $\mathrm{SO}(n, 1)$. If $H$ is any subgroup of $\operatorname{Isom}^{+}\left(\mathbb{H}^{n}\right)$, let $D_{\text {free }}(H)$ denote the set of all numbers $d$ such that $d=\mathcal{H D}(L(F))$, the Hausdorff dimension of the limit set of a free convex cocompact subgroup $F<H$.

Theorem 1.4 If $\Gamma$ is a lattice in $\operatorname{Isom}^{+}\left(\mathbb{H}^{n}\right)$ then $\overline{D_{\text {free }}(\Gamma)}=\overline{D_{\text {free }}\left(\operatorname{Isom}^{+}\left(\mathbb{H}^{n}\right)\right)}$.

This follows immediately from the above theorems.

Remark 1 It is easy and well-known that $D_{\text {free }}\left(\operatorname{Isom}^{+}\left(\mathbb{H}^{2}\right)\right)=(0,1)$. We claim that $D_{\text {free }}\left(\operatorname{Isom}^{+}\left(\mathbb{H}^{3}\right)\right)=(0,2)$. In [14], McMullen proves the following. Let Teich $(S)$ denote the Teichmüller space of a compact connected surface of negative Euler characteristic. We will assume that $S$ has nonempty boundary so that $\pi_{1}(S)$ is free. For every $(X, Y) \in \operatorname{Teich}(S) \times \operatorname{Teich}(S)$ there is discrete convex cocompact group $\Gamma(X, Y)<\operatorname{Isom}^{+}\left(\mathbb{H}^{3}\right)$ isomorphic to $\pi_{1}(S)$ (via the Bers embedding). Moreover, the Hausdorff dimension of the limit set of $\Gamma(X, Y)$ varies continuously (with respect to the Teichmuller metric) [14, Proposition 9.1] and for any $X \in \operatorname{Teich}(S)$, there exists a sequence $X_{i} \in \operatorname{Teich}(S)$ such that the Hausdorff dimension of the limit set of $\Gamma\left(X, X_{i}\right)$ tends to 2 [14, Corollary 9.4]. It is easy to show that there also exists a sequence $X_{i} \in \operatorname{Teich}(S)$ such that the Hausdorff dimension of the limit set of $\Gamma\left(X_{i}, X_{i}\right)$ tends to 0 . Since Teichmüller space is connected, this implies the claim. It is not known whether this result extends to $\operatorname{Isom}^{+}\left(\mathbb{H}^{n}\right)$ for any $n \geq 4$.

A surface group is a group isomorphic to the fundamental group of a closed surface of genus at least 2.

Remark 2 Let $D_{S}(\Gamma)$ denote the set of Hausdorff dimensions of limit sets of surface subgroups of $\Gamma$. In general, $D_{s}(\Gamma)$ is very mysterious. For example, in case $\Gamma<$ $\operatorname{SO}(3,1)$ is a uniform lattice, it is not known whether $\overline{D_{s}(\Gamma)}=[1,2]$ for any $\Gamma$ or for all $\Gamma$ (even for well-studied lattices like the fundamental group of the figure-eight knot complement). It is even unknown whether $\overline{D_{S}(\Gamma)}$ contains an interval for any such $\Gamma$ or for all $\Gamma$. 
Let $P$ be a right-angled, compact Coxeter polyhedron in $\mathbb{H}^{3}$, and let $\Gamma(P) \subset \operatorname{Isom}\left(\mathbb{H}^{3}\right)$ be the group generated by reflections in the faces of $P$.

Theorem 1.5 (Masters [13]) Every finitely generated free subgroup of $\Gamma(P)$ is contained in a subgroup $Q<\Gamma(P)$ isomorphic to the fundamental group of a closed orbifold of negative Euler characteristic.

The corollary below was pointed out by Joseph Masters.

Corollary 1.6 For every $d<2$ there exists a surface subgroup $H_{d}<\Gamma(P)$ such that the Hausdorff dimension of the limit set of $H_{d}$ is at least $d$.

Proof By Remark 1 and Theorem 1.4 there exists a finitely generated free subgroup $F<\Gamma$ with $\mathcal{H} \mathcal{D}(L(F)) \geq d$. By the theorem above, there exists a group $Q$ with $F<Q<\Gamma$ such that $Q$ is isomorphic to the fundamental group of a closed orbifold of negative Euler characteristic. Because $F<Q, \mathcal{H D}(L(Q)) \geq d$. It is well-known that $Q$ contains a finite-index surface subgroup $Q^{\prime}$. Because $Q^{\prime}$ has finite index in $Q$, $\mathcal{H D}\left(L\left(Q^{\prime}\right)\right)=\mathcal{H D}(L(Q)) \geq d$

The next application regards mapping class groups. Let $S$ denote an oriented closed hyperbolic surface and $\operatorname{Mod}(S)=\pi_{0}\left(\operatorname{Homeo}^{+}(S)\right)$ its group of orientation preserving self-homeomorphisms up to isotopy. Let $D_{\text {free }}(S)$ denote the set of all numbers of the form $\mathcal{H} \mathcal{D}(L(F))$, where $F$ is a free convex compact subgroup of $\operatorname{Mod}(S)$ and $\mathcal{H D}(L(F))$ is the Hausdorff dimension of the limit set of $F$ on the boundary at infinity of Teichmüller space. The result below was first observed by Chris Leininger.

Theorem $1.7[0,1] \subset \overline{D_{\text {free }}(S)}$.

Remark 3 It seems highly unlikely that $[0,1]=\overline{D_{\text {free }}(S)}$ but I do not know that this is false.

Proof There exist disks (called Teichmüller disks) contained in Teichmüller space that are isometric with the hyperbolic plane $\mathbb{H}^{2}$. By Veech [18], there exists such a disk whose stabilizer in the mapping class group is a lattice in $\operatorname{PSL}_{2}(\mathbb{R})$, the group of all (orientation-preserving) isometries of $\mathbb{H}^{2}$. The circle at infinity of the hyperbolic plane piecewise projectively embeds in the Thurston boundary of Teichmüller space. So Hausdorff dimension in the circle is the same as in Thurston's boundary with respect to the natural piecewise projective structure. This theorem now follows from Theorem 1.4. 
Theorem 1.4 plays a key role in the two recent papers by Lackenby, Long and Reid [11] and Lackenby [10]. The first relates LERF with the Lubotzky-Sarnak conjecture and the second proves that Kleinian groups that contain noncyclic finite subgroups are either virtually free or contain a surface subgroup.

\subsection{Organization}

To prove Theorem 1.1, the action of $F$ on $\Gamma \backslash G$ is embedded into a symbolic dynamical system over $F$. A result in the symbolic dynamics over a free group implies that this larger system contains a periodic point. That point is used to construct the $\epsilon-$ perturbation $\phi_{\epsilon}$. Thus, the core of the proof is a symbolic dynamics result. That result is stated and proven in Section 2. In Section 3, Theorem 1.1 is proven.

A more general symbolic dynamics result is proven in Section 4. That result is used to prove Theorem 1.2 in Section 5. Section 6 contains the proof of Theorem 1.3. In the last section, we explore attempts to replace free groups with surface groups in Theorem 1.1. We prove that a continuous version of the symbolic dynamics result of Section 2 does not hold. But we conjecture that with an additional hypothesis, the result of Section 2 is true for surface groups. We show that this conjecture implies the surface subgroup conjecture.

Acknowledgements I'd like to thank Joe Masters and Chris Leininger for the applications above. Conversations with Chris Leininger were helpful in formulating the proof of Theorem 1.2. I'm grateful to Marc Lackenby for pointing out several errors in a previous version and making helpful suggestions. I'd like to thank Alan Reid and Darren Long for useful conversations that have improved the paper. Last but not least, the referee has been most helpful by carefully going over the paper and making recommendations.

\section{Symbolic dynamics}

The core of the proof of Theorem 1.1 is a result in the symbolic dynamics over a finitely generated free group $\mathbb{F}$. To describe it, let $S$ be a symmetric free generating set for $\mathbb{F}$. Let $\mathcal{G}=(V, E)$ be a graph. Assume that each edge is directed and has a label in $S . \mathcal{G}$ is allowed to have loops and multiple edges but for each $s \in S$ and vertices $v, w \in V$, we require that there is at most one edge that is labeled $s$ and directed from $v$ to $w$. Such an edge is denoted by the triple $(v, w ; s)$. We will associate to $\mathcal{G}$ a dynamical system over $\mathbb{F}$. 
Let $V^{\mathbb{F}}$ be the set of all functions $x: \mathbb{F} \rightarrow V$ with the topology of uniform convergence on finite sets. Let $X=X_{\mathcal{G}}$ be the set of all $x \in V^{\mathbb{F}}$ such that for all $f \in \mathbb{F}$ and $s \in S$, there is a directed edge in $\mathcal{G}$ from $x(f)$ to $x(f s)$ labeled $s . X$ is called the graph subshift determined by $\mathcal{G} . \mathcal{G}$ is the constraint graph of $X$.

To buttress the analogy with the classical symbolic dynamics over the integers, an element of $V^{\mathbb{F}}$ is called a treequence. For $g \in \mathbb{F}$, the shift operator $\sigma_{g}: V^{\mathbb{F}} \rightarrow V^{\mathbb{F}}$ is defined by $\left(\sigma_{g} x\right)(f)=x\left(g^{-1} f\right)$. This defines an action of $\mathbb{F}$ on $V^{\mathbb{F}} . X_{\mathcal{G}}$ is closed and shift-invariant. Thus $\mathbb{F}$ acts on $X_{\mathcal{G}}$.

The symmetry group of a treequence $x \in X$ is defined by $F_{x}:=\left\{f \in \mathbb{F} \mid \sigma_{f} x=x\right\}$. If it has finite index in $\mathbb{F}$ then $x$ is said to be periodic. A measure $\mu$ on $X$ is shiftinvariant if $\mu\left(\sigma_{g} E\right)=\mu(E)$ for all $g \in \mathbb{F}$ and all measurable sets $E$. Equivalently, $\mu$ is $\mathbb{F}$-invariant.

Theorem 2.1 Let $X \subset V^{\mathbb{F}}$ be the graph subshift defined by a finite graph $\mathcal{G}$. If there exists a shift-invariant Borel probability measure $\mu$ on $X$, then there exists a periodic treequence $x \in X$. Moreover, if for some $v \in V, \mu(\{x \in X \mid x(\mathrm{id})=v\})>0$ then there exists a periodic treequence $x \in X$ with $x$ (id) $=v$. Here, id denotes the identity element.

Remark 4 This theorem is the only place in the proof of Theorem 1.1 where the fact that $\mathbb{F}$ is a free group is used. Theorem 2.1 is not true if $\mathbb{F}$ is replaced by the fundamental group of a closed surface of genus at least 2 . However, it is possible that under additional hypotheses on $X$, it remains true. See Section 7 for further discussion.

Remark 5 If $\mathbb{F}$ has rank at least 2 then, because $\mathbb{F}$ is nonamenable, it is possible that there are no shift-invariant Borel probability measures on $X$.

The proof of Theorem 2.1 given next is essentially the same as the proof given in [1], where it was introduced. The idea comes from a simple observation: if $x: \mathbb{F} \rightarrow V$ is periodic, then it descends to a function $\bar{x}: F_{x} \backslash \mathbb{F} \rightarrow V$ by $\bar{x}\left(F_{x} f\right)=x(f)$. To prove Theorem 2.1, we will construct a periodic treequence by reversing this procedure. That is, we will construct a right action of $\mathbb{F}$ on a finite set $K$ and a function $\bar{x}: K \rightarrow V$ such that some "lift" of this function (defined by $x(f)=\bar{x}\left(k_{1} \cdot f\right)$ where $k_{1} \in K$ is fixed), is a periodic treequence in $X$. This is accomplished through a study of weights on the constraint graph $\mathcal{G}$ which are defined next.

Let $V=V(\mathcal{G}), E(\mathcal{G})$ denote the vertex set and edge set of $\mathcal{G}$ respectively. We write $(v, w ; s)$ to denote the edge in $E(\mathcal{G})$ from $v$ to $w$ labeled $s$ (where $v, w \in V, s \in S$ ) 
if one exists. A weight on $\mathcal{G}$ is a function $W: V(\mathcal{G}) \cup E(\mathcal{G}) \rightarrow[0, \infty)$ such that

$$
\begin{aligned}
\forall v \in V(\mathcal{G}), s \in S, & W(v)=\sum_{w \in V} W(v, w ; s)=\sum_{w \in V} W(w, v ; s), \\
\forall v, w \in V(\mathcal{G}), s \in S, & W(v, w ; s)=W\left(w, v ; s^{-1}\right) .
\end{aligned}
$$

The first sum above is over all $w \in V$ such that $(v, w ; s) \in E(\mathcal{G})$ and the second sum is over all $w \in V$ such that $(w, v ; s) \in E(\mathcal{G})$. A weight is nontrivial if it is not identically zero.

Lemma 2.2 Let $\mu$ be a shift-invariant Borel probability measure on $X$. Let

$$
\begin{aligned}
W_{\mu}(v) & =\mu(\{x \in X \mid x(\mathrm{id})=v\}), \\
W_{\mu}(v, w ; s) & =\mu(\{x \in X \mid x(\mathrm{id})=v, x(s)=w\}) .
\end{aligned}
$$

Then $W_{\mu}$ is a nontrivial weight on $\mathcal{G}$.

Proof The following equation holds by additivity of $\mu$ :

$$
W_{\mu}(v)=\sum_{w \in V} W_{\mu}(v, w ; s)=\sum_{w \in V} W_{\mu}(w, v ; s) .
$$

The equation $W_{\mu}(v, w ; s)=W_{\mu}\left(w, v ; s^{-1}\right)$ is true because $\mu$ is shift-invariant and $\sigma_{s}(\{x \in X \mid x(\mathrm{id})=v, x(s)=w\})=\left\{x \in X \mid x(\mathrm{id})=w, x\left(s^{-1}\right)=v\right\}$.

Lemma 2.3 Let $W: V(\mathcal{G}) \cup E(\mathcal{G}) \rightarrow[0, \infty)$ be a nontrivial weight. If, for some $v_{1} \in V(\mathcal{G}), W\left(v_{1}\right)>0$, then there exists a periodic treequence $x \in X$ with $x$ (id) $=v_{1}$.

Proof The weight equations (1)-(2) are linear equations. So the space of all weights on $\mathcal{G}$ is the intersection of a certain linear subspace of $\mathbb{R}^{V(\mathcal{G}) \cup E(\mathcal{G})}$ with the positive orthant. Because these equations have coefficients in $\mathbb{Z}$, the existence of the weight $W$ with $W\left(v_{1}\right)>0$ implies the existence of a rational weight $W^{\prime}$ such that $W^{\prime}\left(v_{1}\right)>0$. Rational means that $W^{\prime}(v)$ and $W^{\prime}(e)$ are rational numbers for every $v \in V(\mathcal{G})$ and $e \in E(\mathcal{G})$. In fact, we may assume that $W^{\prime}(v)$ and $W^{\prime}(e)$ are integers for every $v \in V(\mathcal{G})$ and $e \in E(\mathcal{G})$ since multiplying a weight by a positive scalar does not change the fact that it is a weight.

Since $S$ is a symmetric free generating set for $\mathbb{F}$, we may write $S=\left\{s_{1}, \ldots, s_{r}\right.$, $\left.s_{1}^{-1}, \ldots, s_{r}^{-1}\right\}$. Let $S^{+}=\left\{s_{1}, \ldots, s_{r}\right\}$. So $\mathbb{F}=\left\langle s_{1}, \ldots, s_{r}\right\rangle$.

By the above, we may assume that $W(v)$ and $W(e)$ are integers for every $v \in V(\mathcal{G})$ and $e \in E(\mathcal{G})$. For each $v \in V(\mathcal{G})$, let $K(v)$ be a set with $|K(v)|=W(v)$. For every $v \in V(\mathcal{G})$ and $s \in S^{+}$, choose a partition $\left\{K_{+}(v, w ; s)\right\}_{w \in V}$ of $K(v)$ so that 
$\left|K_{+}(v, w ; s)\right|=W(v, w ; s)$. This is possible because of the weight equations (1)-(2). Similarly, choose a partition $\left\{K_{-}(v, w ; s)\right\}_{w \in V}$ of $K(v)$ so that $\left|K_{-}(v, w ; s)\right|=$ $W\left(w, v ; s^{-1}\right)$. For each $v, w \in V$ and $s \in S^{+}$, choose a bijection $b_{v, w ; s}: K_{+}(v, w ; s) \rightarrow$ $K_{-}(w, v ; s)$.

Let $K$ be the disjoint union $\bigsqcup_{v \in V} K(v)$. For $s \in S^{+}$, the bijections $\left\{b_{v, w ; s}\right\}_{v, w \in V}$ form a permutation of $K$ as follows. For $k \in K(v)$, let $k \cdot s:=b_{v, w ; s}(k)$ where $w \in V$ is the unique element such that $k \in K_{+}(v, w ; s)$. Since $S$ freely generates $\mathbb{F}$ as a group, this defines a right action of $\mathbb{F}$ on $K$.

Let $\bar{x}: K \rightarrow V(\mathcal{G})$ be the function $\bar{x}(k)=v$ if $k \in K(v)$. Now we can choose a periodic treequence $x \in X$ as follows. Let $k_{1} \in K_{v_{1}}$. For $f \in \mathbb{F}$, define $x(f)=\bar{x}\left(k_{1} \cdot f\right)$. Because $K$ is finite, the stabilizer $F_{k_{1}}:=\left\{f \in \mathbb{F} \mid k_{1} \cdot f=k_{1}\right\}$ has finite index in $\mathbb{F}$. Since $\sigma_{f} x=x$ for every $f \in F_{k_{1}}, x$ is periodic. Also $x$ (id) $=\bar{x}\left(k_{1}\right)=v_{1}$. Apriori, $x$ is only in $V^{\mathbb{F}}$. Let us check that $x \in X$. Let $f \in F, s \in S^{+}$. Let $l=k_{1} \cdot f$, $v=x(f)=\bar{x}(l)$ and $w=x(f \cdot s)=\bar{x}(l \cdot s)$. Then $b_{v, w ; s}(l)=l \cdot s$. Thus $W(v, w ; s)>0$ which implies $(v, w ; s) \in E(\mathcal{G})$. So $x \in X$.

Theorem 2.1 is an immediate consequence of the lemmas above.

\section{The uniform case}

In this section, Theorem 1.1 is proven. So all the hypotheses of Theorem 1.1 are assumed. Briefly, the proof goes as follows. $\mathbb{F}$ acts on $\Gamma \backslash G$ on the left by $f(\Gamma g):=\Gamma g \phi\left(f^{-1}\right)$. The space $\Gamma \backslash G$ is partitioned into subsets of small diameter. This partitioning is used to measurably embed $\Gamma \backslash G$ into a graph subshift $X$. Since $G$ is unimodular, Haar measure on $G$ descends to a $G$-invariant Haar measure on $\Gamma \backslash G$. This pushes forward to a shift-invariant measure on $X$. Theorem 2.1 implies the existence of a periodic treequence $x \in X$. This treequence is "decoded" to produce the required $\epsilon$-perturbation.

\subsection{The graph subshift}

Consider $\Gamma \backslash G$ with the quotient metric $\bar{d}$ given by $\bar{d}\left(\Gamma g_{1}, \Gamma g_{2}\right)=\min _{\gamma \in \Gamma} d\left(\gamma g_{1}, g_{2}\right)$. Recall that $S$ is a symmetric free generating set for $\mathbb{F}$. Let $\delta>0$ be such that for all $g_{1}, g_{2} \in G$ with $d\left(g_{1}\right.$, id $)<\delta$ and $d\left(g_{2}\right.$,id $)<\delta$, if $s \in S$ then

$$
d\left(g_{1} \phi(s) g_{2}, \phi(s)\right)<\epsilon .
$$

Let $V=\left\{v_{1}, v_{2}, \ldots, v_{n}\right\}$ be a Borel partition of $\Gamma \backslash G$ into sets $v_{i}$ of diameter less than $\delta$. Assume that each $v_{i}$ has positive Haar measure. Let $\mathcal{G}$ be the graph with vertex 
set $V=\left\{v_{1}, \ldots, v_{n}\right\}$ and edges defined as follows. For each $v, w \in V$, if there exist elements $p \in v, q \in w$ and $s \in S$ such that $p \phi(s)=q$ then there is a directed edge in $\mathcal{G}$ from $v$ to $w$ labeled $s$ which we denote by $(v, w ; s)$. There are no other edges.

Let $X \subset V^{\mathbb{F}}$ be the graph subshift determined by $\mathcal{G}$.

\subsection{Perturbations from treequences}

We will choose, for each $x \in X$, an $\epsilon$-perturbation $\phi_{x}$ of $\phi$. To get started, choose a basepoint $p_{i} \in v_{i}$ for each $i$. Without loss of generality, assume $p_{1}=\Gamma$.

If there is an edge $e=(v, w ; s)$ in $\mathcal{G}$ then there exist points $p \in v, q \in w$ such that $p \phi(s)=q$. Let $p_{v}, q_{w}$ be the basepoints of $v$ and $w$ respectively. Because $v$ and $w$ each have diameter at most $\delta$, there exists elements $g_{v}, g_{w} \in G$ such that $d\left(g_{v}, \mathrm{id}\right)<\delta, d\left(g_{w}, \mathrm{id}\right)<\delta, p_{v} g_{v}=p$ and $q_{w} g_{w}=q$. Let $\psi_{e}=g_{v} \phi(s) g_{w}^{-1}$. Note that $p_{v} \psi_{e}=p_{v} g_{v} \phi(s) g_{w}^{-1}=q_{w}$. By choice of $\delta, d\left(\psi_{e}, \phi(s)\right)<\epsilon$.

There is also an edge $e^{\prime}=\left(w, v: s^{-1}\right)$ in $\mathcal{G}$. We choose $\psi_{e^{\prime}}=\psi_{e}^{-1}$, and observe that $q_{w} \psi_{e^{\prime}}=p_{v}$ and $d\left(\psi_{e^{\prime}}, \phi\left(s^{-1}\right)\right)<\epsilon$.

Let $x \in X$. For $f \in \mathbb{F}$, represent $f$ as $f=t_{1} \cdots t_{m}$ for some $t_{i} \in S$. Let $t_{0}=\mathrm{id}$. Let $\phi_{x}(f)=\psi_{e_{1}} \cdots \psi_{e_{m}}$ where

$$
e_{i}=\left(x\left(t_{0} \cdots t_{i-1}\right), x\left(t_{1} \cdots t_{i}\right) ; t_{i}\right) .
$$

This is independent of the choice of representation of $f$ because of the choice for $\psi_{e^{\prime}}$ above and because $\mathbb{F}$ is freely generated by $S$.

That $\phi_{x}$ is an $\epsilon$-perturbation of $\phi$ follows from the next lemma.

Lemma 3.1 A map $\eta: \mathbb{F} \rightarrow G$ is an $\epsilon$-perturbation of $\phi$ if for any sequence $t_{1}, \ldots$, $t_{m} \in S$, there exist elements $t_{i}^{\prime} \in G$ such that $d\left(\phi\left(t_{i}\right), t_{i}^{\prime}\right)<\epsilon$ and $\eta\left(t_{1} \cdots t_{i}\right)=t_{1}^{\prime} \cdots t_{i}^{\prime}$ for all $i=1, \ldots, m$.

Proof Let $f \in \mathbb{F}$ and $s \in S$. Then there exists a sequence $t_{1}, \ldots, t_{m} \in S$ such that $f=t_{1} \cdots t_{m}$. Let $t_{m+1}=s$. By hypothesis, for $1 \leq i \leq m+1$, there exist elements $t_{i}^{\prime} \in G$ such that $d\left(\phi\left(t_{i}\right), t_{i}^{\prime}\right)<\epsilon$ and $\eta\left(t_{1} \cdots t_{i}\right)=t_{1}^{\prime} \cdots t_{i}^{\prime}$ for all $i=1, \ldots, m+1$. Thus

$$
\begin{aligned}
d(\eta(f s), \eta(f) \phi(s)) & =d\left(t_{1}^{\prime} \cdots t_{m+1}^{\prime}, t_{1}^{\prime} \cdots t_{m}^{\prime} \phi(s)\right) \\
& =d\left(t_{m+1}^{\prime}, \phi(s)\right)=d\left(t_{m+1}^{\prime}, \phi\left(t_{m+1}\right)\right)<\epsilon .
\end{aligned}
$$

Corollary 3.2 For every $x \in X$, the map $\phi_{x}: \mathbb{F} \rightarrow G$ is an $\epsilon$-perturbation of $\phi$. 


\subsection{Embedding $\Gamma \backslash G$ in $X$}

Let $L: \Gamma \backslash G \rightarrow V$ be the labeling map. That is, $L(\Gamma g)=v$ if $\Gamma g \in v$. For each $\Gamma g \in \Gamma \backslash G$, let $\Psi(\Gamma g) \in V^{\mathbb{F}}$ be the treequence $\Psi(\Gamma g)(f)=L(\Gamma g \phi(f))$.

$\mathbb{F}$ acts on the left on $\Gamma \backslash G$ by: $f \cdot \Gamma g:=\Gamma g \phi\left(f^{-1}\right)$. This action preserves $\mu$, the normalized Haar measure on $\Gamma \backslash G . \Psi$ is equivariant with respect to the left actions of $\mathbb{F}$. The image of $\Psi$ lies inside $X$ by definition, so $\Psi_{*}(\mu)$ is a shift-invariant Borel probability measure on $X$. By Theorem 2.1, there exists a periodic treequence $z \in X$. Indeed, since $\mu\left(v_{1}\right)>0$,

$$
\Psi_{*}(\mu)\left(\left\{x \in X \mid x(\mathrm{id})=v_{1}\right\}\right)>0 .
$$

Thus, there exists a periodic treequence $z \in X$ such that $z(\mathrm{id})=v_{1}$.

To finish the proof of Theorem 1.1, we claim that $\phi_{z}$ is virtually a homomorphism into $\Gamma$. We will need the following lemma.

Lemma 3.3 For any $x \in X$ and $f, g \in \mathbb{F}, \phi_{x}(f) \phi_{f^{-1} x}(g)=\phi_{x}(f g)$.

Proof Let $f=t_{1} \cdots t_{m}, g=u_{1} \cdots u_{k}$ with $t_{i}, u_{i} \in S$. Let $t_{0}=u_{0}=$ id. Let

$$
\begin{aligned}
e_{i} & :=\left(x\left(t_{0} \cdots t_{i-1}\right), x\left(t_{1} \cdots t_{i}\right) ; t_{i}\right), \\
e_{j}^{\prime} & :=\left(x\left(f u_{0} \cdots u_{j-1}\right), x\left(f u_{1} \cdots u_{j}\right) ; u_{j}\right) .
\end{aligned}
$$

By definition, $\phi_{x}(f)=\psi_{e_{1}} \cdots \psi_{e_{m}}$ and $\phi_{x}(f g)=\psi_{e_{1}} \cdots \psi_{e_{m}} \psi_{e_{1}^{\prime}} \cdots \psi_{e_{k}^{\prime}}$.

Note that

$$
e_{i}^{\prime}=\left(\left(f^{-1} x\right)\left(u_{0} \cdots u_{i-1}\right),\left(f^{-1} x\right)\left(u_{1} \cdots u_{i}\right) ; u_{i}\right) .
$$

Therefore, $\phi_{f^{-1} x}(g)=\psi_{e_{1}^{\prime}} \cdots \psi_{e_{k}^{\prime}}=\phi_{x}(f)^{-1} \phi_{x}(f g)$.

Now, let $F_{z}<\mathbb{F}$ be the symmetry group of $z$. Since $z$ is periodic, $F_{z}$ has finite index in $\mathbb{F}$. Let $f \in F_{z}$ and $g \in \mathbb{F}$. Then

$$
\phi_{z}(f g)=\phi_{z}(f) \phi_{f^{-1} z}(g)=\phi_{z}(f) \phi_{z}(g) .
$$

This shows that $\phi_{z}$ is virtually a homomorphism. To show that $\phi_{z}\left(F_{z}\right)<\Gamma$, we need the next lemma.

Lemma 3.4 Let $x \in X$ be such that $x(\mathrm{id})=v_{1}$. Then, for any $f \in \mathbb{F}, \Gamma \phi_{x}(f)$ is the basepoint of $x(f) \in V$. 
Proof Let $f=t_{1} \cdots t_{m}$ with $t_{i} \in S$. Let $t_{0}=$ id. Let

$$
e_{i}:=\left(x\left(t_{0} \cdots t_{i-1}\right), x\left(t_{1} \cdots t_{i}\right) ; t_{i}\right) .
$$

By definition, $\phi_{x}(f)=\psi_{e_{1}} \cdots \psi_{e_{m}}$.

Let $q_{i}$ be the basepoint of $x\left(t_{0} \cdots t_{i}\right) \in V$. By definition of $\psi_{e}, q_{i-1} \psi_{e_{i}}=q_{i}$. So $q_{0} \phi_{x}(f)=q_{m}$. Since $x(\mathrm{id})=v_{1}, q_{0}=\Gamma$. So $\Gamma \phi_{x}(f)=q_{m}$ is the basepoint of $x\left(t_{0} \cdots t_{m}\right)=x(f)$.

The lemma implies that for $f \in F_{z}, \Gamma \phi_{z}(f)$ is the basepoint of $z(f) \subset \Gamma \backslash G$. But $z(f)=z(\mathrm{id})=v_{1}$ since $f \in F_{z}$. So the basepoint of $z(f)$ is $\Gamma$. That is, $\Gamma \phi_{z}(f)=\Gamma$. Of course, this implies $\phi_{z}\left(F_{z}\right)<\Gamma$. This completes the proof of Theorem 1.1.

\section{Subshifts determined by infinite graphs}

The proof of Theorem 1.2 follows the same ideas as the proof of Theorem 1.1. However, because $\Gamma \backslash G$ may be noncompact, it is necessary to work with infinite partitions and therefore, with subshifts determined by infinite graphs. Theorem 2.1 does not apply in this case. So we generalize Theorem 2.1 to certain infinite-graph subshifts. This is used in the next section to prove Theorem 1.2. To begin, we need some definitions.

Definition 1 Recall that $S \subset \mathbb{F}$ is a finite symmetric free generating set. Let $\mathcal{F}$ be the Cayley graph of $\mathbb{F}$. It has vertex set $\mathbb{F}$ and for every $f \in \mathbb{F}$ and $s \in S$, there is a directed edge from $f$ to $f s$ labeled $s$. The induced subgraph of a set $F \subset \mathbb{F}$ is the largest subgraph of $\mathcal{F}$ with vertex set $F$. If it connected then we say $F$ is $S$-connected. An $S$-connected component of a set $F \subset \mathbb{F}$ is an $S$-connected subset $D \subset \mathbb{F}$ that is maximal among all $S$-connected subsets of $F$ with respect to inclusion.

Theorem 4.1 Let $X \subset V^{\mathbb{F}}$ be a graph subshift determined by a graph $\mathcal{G}=(V, E)$. Suppose that there is a finite set $A \subset V$ and a shift-invariant Borel probability measure $\mu$ on $X$ such that for $\mu$-almost every $x \in X$, every $S$-connected component the of set $x^{-1}(V-A) \subset \mathbb{F}$ is finite. Then there exists a periodic treequence in $X$. If for some $a_{1} \in A, \mu\left(\left\{x \in X \mid x(\mathrm{id})=a_{1}\right\}\right)>0$, then there exists a periodic treequence $x \in X$ with $x(\mathrm{id})=a_{1}$.

The rest of this section proves this theorem. The next section shows how to apply this result to obtain Theorem 1.2. To prove this theorem, we show that there exists a weight supported on a finite subgraph of $\mathcal{G}$ and then invoke Lemma 2.3. To do this, we represent the weight $W_{\mu}$ as a sum of functions that correspond to $x^{-1}(A)$ and the 
connected components of $x^{-1}(V-A)$ for $x \in X$. Then a simple convex geometric argument yields the existence of the desired weight. We will need some definitions.

Let $W_{\mu}$ be as defined in Lemma 2.2. Let $W_{\mu}^{\prime}: V \cup E \rightarrow[0, \infty)$ be the function defined by "truncating $W_{\mu}$ " off of $V-A$. To be precise:

- $W_{\mu}^{\prime}(a)=W_{\mu}(a)$ for $a \in A$,

- $W_{\mu}^{\prime}(a, b ; s)=W_{\mu}(a, b ; s)$ for $a, b \in A$ and $s \in S$,

- $W_{\mu}^{\prime}(v)=0$ for $v \in V-A$,

- $W_{\mu}^{\prime}(v, w ; s)=0$ if either $v \in V-A$ or $w \in V-A$.

$W_{\mu}^{\prime}$ is not a weight in general. We will write $W_{\mu}$ as a sum of $W_{\mu}^{\prime}$ and some other functions, defined next.

The outer boundary of a set $C \subset \mathbb{F}$ is the set of all elements $f \in \mathbb{F}$ such that $f \notin C$ but $f$ is adjacent to an element in $C$ (ie, $\exists s \in S$ such that $f s \in C$ ). It is denoted by $\partial_{o} C$.

Let $Z$ be the collection of all functions $z: D_{z} \rightarrow V$ such that

- the domain of $z$, denoted $D_{z} \subset \mathbb{F}$, is finite and

- if $C_{z}=z^{-1}(V-A)$ then $C_{z}$ is $S$-connected and $D_{z}=C_{z} \cup \partial_{o} C_{z}$.

For $z \in Z$, let $[z] \subset V^{\mathbb{F}}$ be the set of all functions $x: \mathbb{F} \rightarrow V$ such that there is an $f \in \mathbb{F}$ satisfying

- $f^{-1} \in C_{z}$,

- $x(f d)=z(d)$ for all $d \in D_{z}$.

Recall that $(v, w ; s)$ denotes the edge in $E(\mathcal{G})$ from $v$ to $w$ labeled $s$ (where $v, w \in V$ and $s \in S)$ if one exists. For $z \in Z$, define a function $W_{z}: V(\mathcal{G}) \cup E(\mathcal{G}) \rightarrow[0, \infty)$ as follows.

- For $a \in A$, let $W_{z}(a)=0$.

- For $v \in V-A$, let

$$
W_{z}(v)=\mu(\{x \in X \mid x(\mathrm{id})=v, x \in[z]\}) .
$$

- For $v \in V-A, w \in V$ and $s \in S$, let

$$
W_{z}(v, w ; s)=\mu(\{x \in X \mid x(\mathrm{id})=v, x(s)=w, x \in[z]\}) .
$$

- For $a \in A, v \in V-A, s \in S$ let $W_{z}(a, v ; s)=W_{z}\left(v, a ; s^{-1}\right)$.

- Let $W_{z}(a, b ; s)=0$ for any $a, b \in A$ and $s \in S$. 
The function $W_{z}$ is not a weight in general. Since the domain of each $z \in Z$ is finite, $W_{z}$ is supported on a finite subgraph of $\mathcal{G}$ (ie, the subset of $V \cup E$ on which $W_{z}$ is nonzero is finite). Choose a subcollection $Z^{\prime} \subset Z$ such that for all $z \in Z$ there exists a unique $z^{\prime} \in Z^{\prime}$ with $[z]=\left[z^{\prime}\right]$. Note that for any $z_{1}, z_{2} \in Z$ either $\left[z_{1}\right]=\left[z_{2}\right]$ or $\left[z_{1}\right] \cap\left[z_{2}\right]=\varnothing$. Therefore, $\left\{\left[z^{\prime}\right]: z^{\prime} \in Z^{\prime}\right\}$ is a partition of the set of all $x \in V^{\mathbb{F}}$ such that $x$ (id) $\in V-A$ and the $S$-connected component of $x^{-1}(V-A)$ containing id is finite.

\section{Lemma 4.2}

$$
W_{\mu}=W_{\mu}^{\prime}+\sum_{z \in Z^{\prime}} W_{z}
$$

Proof This follows immediately from the definitions and the hypothesis on $\mu$ (namely that $\mu$ is supported on the set of all $x \in V^{\mathbb{F}}$ such that either $x$ (id) $\in A$ or $x$ (id) $\in V-A$ and the $S$-connected component of $x^{-1}(V-A)$ containing id is finite).

Let $\vec{v} \in \mathbb{R}^{A \times S}$ be the vector

$$
\vec{v}(a, s)=\sum_{b \in V-A} W_{\mu}(a, b ; s) .
$$

For $z \in Z$, let $\vec{v}_{z} \in \mathbb{R}^{A \times S}$ be the vector

$$
\vec{v}_{z}(a, s)=\sum_{b \in V-A} W_{z}(a, b ; s) .
$$

The lemma above implies $\vec{v}(a, s)=\sum_{z \in Z^{\prime}} \vec{v}_{z}(a, s)$. The next lemma enables us to replace this sum with a finite sum.

Lemma 4.3 Let $R=\left\{\vec{r}_{i}\right\}_{i=1}^{\infty}$ be a sequence of nonnegative vectors in $\mathbb{R}^{k}$ for some $k<\infty$. Let $\vec{r}_{\infty}$ be the sum $\vec{r}_{\infty}:=\sum_{i=1}^{\infty} \vec{r}_{i}$. If $\vec{r}_{\infty} \in \mathbb{R}^{k}$ (ie, every component of $\vec{r}_{\infty}$ is finite) then there exists an $N>0$ and nonnegative coefficients $t_{1}, \ldots, t_{N}$ such that $\vec{r}_{\infty}=\sum_{i=1}^{N} t_{i} \vec{r}_{i}$

Proof If $U$ is a set of vectors in $\mathbb{R}^{k}$, then the positive cone of $U$ is the set of all vectors that can be expressed as $\sum_{i=1}^{\infty} c_{i} u_{i}$ with $c_{i} \geq 0$ and $u_{i} \in U$. Let $C$ be the closure of the positive cone of $R=\left\{\vec{r}_{i}\right\}_{i=1}^{\infty}$.

If the interior of $C$ is empty, then $C$ lies inside some linear subspace of $\mathbb{R}^{k}$ of positive codimension in which $C$ has nonempty interior. After replacing $\mathbb{R}^{k}$ with this subspace if necessary, it may be assumed that the interior of $C$ is nonempty.

Claim $1 \vec{r}_{\infty}$ is in the interior of $C$. 
Proof Suppose for a contradiction that $\vec{r}_{\infty}$ is on the boundary of $C$. Because $C$ is convex, there exists a supporting hyperplane $\Pi$ to $C$ at $\vec{r}_{\infty}$. So, $\vec{r}_{\infty} \in \Pi$ and $C$ lies in one of the closed halfspaces determined by $\Pi$. Since the interior of $C$ is nonempty, there exists $\vec{r}_{j} \in R$ such that $\vec{r}_{j} \notin \Pi$.

Since $\vec{r}_{j} \notin \Pi$ and $\vec{r}_{\infty} \in \Pi$, it follows that the vector $\vec{r}_{\infty}-\vec{r}_{j}$ lies in the open half-space determined by $\Pi$ that does not contain $\vec{r}_{j}$, ie, the half-space that does not contain the interior of $C$. But $\vec{r}_{\infty}-\vec{r}_{j}=\sum_{i \neq j} \vec{r}_{i}$ is contained in $C$. This contradiction proves the claim.

Let $C_{n}$ be the positive cone of $\left\{\vec{r}_{1}, \ldots, \vec{r}_{n}\right\}$.

Claim 2 If $\vec{w}$ is any point in the interior of $C$, then there exists $N>0$ such that $\vec{w} \in C_{N}$.

Proof Suppose for a contradiction that $\vec{w}$ is not in $C_{n}$ for any $n$. Because $C_{n}$ is convex there exists a hyperplane $\Pi_{n}$ containing $\vec{w}$ that has trivial intersection with $C_{n}$. The sequence $\left\{\Pi_{n}\right\}$ has a subsequential limit hyperplane $\Pi$ (with respect to the Hausdorff topology). Because $\left\{C_{n}\right\}$ is an increasing sequence of convex sets, it follows that $\Pi$ does not intersect any of the $C_{n}$ 's. Therefore $C$ must be contained in one of the closed half-spaces determined by $\Pi$. But this contradicts the hypothesis that $\vec{w}$ is in the interior of $C$.

The two claims imply the lemma.

By the lemma, there exists a finite collection $Z^{\prime \prime} \subset Z^{\prime}$ and nonnegative coefficients $t_{z}$ (for $z \in Z^{\prime \prime}$ ) such that $\vec{v}=\sum_{z \in Z^{\prime \prime}} t_{z} \vec{v}_{z}$. Define a function $W$ on $\mathcal{G}$ by $W=$ $W_{\mu}^{\prime}+\sum_{z \in Z^{\prime \prime}} t_{z} W_{z}$.

Lemma 4.4 $W$ is weight on $\mathcal{G}$. It is supported on a finite subgraph. If for some $a_{1} \in A, W_{\mu}\left(a_{1}\right)>0$ then $W\left(a_{1}\right)>0$.

Proof It is immediate from the definitions that $W(v, w ; s)=W\left(w, v ; s^{-1}\right)$ for all $v, w \in V$ and $s \in S$. We must show that Equation (1) holds for $W$. This is accomplished in two separate cases.

Case 1 Let $a \in A$ and $s \in S$. We must show that $W(a)=\sum_{b \in V} W(a, b ; s)$. First,

$$
\begin{aligned}
\sum_{b \in V-A} W(a, b ; s) & =\sum_{b \in V-A} \sum_{z \in Z^{\prime \prime}} t_{z} W_{z}(a, b ; s) \\
& =\sum_{z \in Z^{\prime \prime}} t_{z} \vec{v}_{z}(a, s)=\vec{v}(a, s)=\sum_{b \in V-A} W_{\mu}(a, b ; s) .
\end{aligned}
$$


If $b \in A$ then $W(a, b ; s)=W_{\mu}^{\prime}(a, b ; s)=W_{\mu}(a, b ; s)$. So,

$$
\begin{aligned}
W(a)=W_{\mu}(a) & =\sum_{b \in A} W_{\mu}(a, b ; s)+\sum_{b \in V-A} W_{\mu}(a, b ; s) \\
& =\sum_{b \in A} W(a, b ; s)+\sum_{b \in V-A} W(a, b ; s)=\sum_{b \in V} W(a, b ; s) .
\end{aligned}
$$

Case 2 Let $v \in V-A$ and $s \in S$. We must show that $W(v)=\sum_{w \in V} W(v, w ; s)$. For any $z \in Z$,

$$
\begin{aligned}
W_{z}(v) & =\mu(\{x \in X \mid x(\mathrm{id})=v, x \in[z]\}) \\
& =\sum_{w \in V} \mu(\{x \in X \mid x(\mathrm{id})=v, x(s)=w, x \in[z]\})=\sum_{w \in V} W_{z}(v, w ; s) .
\end{aligned}
$$

Thus, $W(v)=\sum_{z \in Z^{\prime \prime}} t_{z} W_{z}(v)=\sum_{z \in Z^{\prime \prime}} t_{z} \sum_{w \in V} W_{z}(v, w ; s)=\sum_{w \in V} W(v, w ; s)$.

From Cases 1 and 2 and the fact that $W(v, w ; s)=W\left(w, v ; s^{-1}\right)$, it follows that for any $v \in V$ and $s \in S$,

$$
W(v)=\sum_{w \in V} W(v, w ; s)=\sum_{w \in V} W\left(w, v ; s^{-1}\right) .
$$

Thus $W$ is a weight. It is supported on a finite subgraph because $Z^{\prime \prime}$ is finite and each $W_{z}$ for $z \in Z^{\prime \prime}$ has finite support.

Theorem 4.1 now follows from the lemma above and Lemma 2.3.

\section{The nonuniform case}

The key ingredient to proving Theorem 1.2 from Theorem 4.1 is the next lemma. Fix a nonuniform lattice $\Gamma<G=\operatorname{SO}(n, 1)=\operatorname{Isom}^{+}\left(\mathbb{H}^{n}\right)$. After passing to a finite index subgroup, we may assume, by Selberg's lemma, that $\Gamma$ is torsion-free. So $\mathbb{H}^{n} / \Gamma$ is a manifold. Let $\phi: \mathbb{F} \rightarrow G$ be an injective homomorphism onto a convex cocompact subgroup of $G$. Note that $\operatorname{SO}(n, 1)$ is unimodular, so Haar measure on $G$ induces a $G$-invariant probability measure on $\Gamma \backslash G$.

Lemma 5.1 For any $\delta>0$ there exists a Borel partition $V=\left\{v_{1}, v_{2}, \ldots\right\}$ of $\Gamma \backslash G$ into sets of diameter at most $\delta$ and a finite set $A \subset V$ such that the following holds.

Let $L: \Gamma \backslash G \rightarrow V$ be the labeling map. So $L(\Gamma g)=v$ if $\Gamma g \in v$. For each $\Gamma g \in \Gamma \backslash G$, let $\Psi(\Gamma g) \in V^{\mathbb{F}}$ be the treequence $\Psi(\Gamma g)(f)=L(\Gamma g \phi(f))$. Let $\mu$ be the probability 
measure on $\Gamma \backslash G$ induced by Haar measure on $G$. Then for $\mu$-almost every $\Gamma g \in \Gamma \backslash G$, every $S$-connected component of $\Psi(\Gamma g)^{-1}(V-A)$ is finite. (See the previous section for the definition of $S$-connected).

Proof Let $C \subset \partial \mathbb{H}^{n}$ be the cusp set of $\Gamma$. That is, $C$ is the set of all points $c \in \partial \mathbb{H}^{n}$ such that there exists a nontrivial parabolic element $g \in \Gamma$ with $g c=c$. It is countable since $\Gamma$ is countable.

Identify $\mathbb{H}^{n}$ with $G / K$ where $K<G$ is a maximal compact subgroup. Then $\mathbb{H}^{n} / \Gamma$ is identified with $\Gamma \backslash G / K$. Let $Q: \mathbb{H}^{n} \rightarrow \mathbb{H}^{n} / \Gamma$ be the quotient map. By Margulis' thin/thick decomposition of $\mathbb{H}^{n} / \Gamma$, there exists a compact set $T \subset \mathbb{H}^{n} / \Gamma$ such that if $T^{c}$ denotes the complement of $T$ in $\mathbb{H}^{n} / \Gamma$ then every connected component of $Q^{-1}\left(T^{c}\right)$ is a horoball. Each of these horoballs has a unique limit point $l \in \partial \mathbb{H}^{n}$ that is contained in the cusp set $C$.

Choose a set $T$ as above so that $\Gamma K \in T$. Also choose $T$ so that for any $g \in G$ and $s \in S, \Gamma g K$ and $\Gamma g \phi(s) K$ cannot be in different connected components of $T^{c}$. To accomplish this, let $d_{\Gamma}$ be the distance function on $\mathbb{H}^{n} / \Gamma$ given by

$$
d_{\Gamma}\left(\Gamma g_{1} K, \Gamma g_{2} K\right)=\min \left\{d\left(\gamma_{1} g_{1} k_{1}, \gamma_{2} g_{2} k_{2}\right) \mid \gamma_{1}, \gamma_{2} \in \Gamma, k_{1}, k_{2} \in K\right\} .
$$

Choose $T$ so large so that if $p, q$ are in different components of $T^{c}$ then $d_{\Gamma}(p, q)>$ $\max _{s \in S} d(\phi(s)$, id $)$. Then for any $g \in G, d_{\Gamma}(\Gamma g K, \Gamma g \phi(s) K)<d(\phi(s)$, id $)$. Thus $\Gamma g K$ and $\Gamma g \phi(s) K$ cannot be in different components of $T^{c}$.

Let $\pi: \Gamma \backslash G \rightarrow \mathbb{H}^{n} / \Gamma$ be the projection map. Choose a Borel partition $V=\left\{v_{1}, v_{2}, \ldots\right\}$ of $\Gamma \backslash G$ into sets of diameter at most $\delta$ so that for some $A \subset V, \pi^{-1}(T)=\bigcup_{a \in A} a$.

Claim If $g \in G$ is such that some $S$-connected component of $\Psi(\Gamma g)^{-1}(V-A)$ is infinite then $g L(\phi(\mathbb{F})) \cap C \neq \varnothing$.

Proof If some $S$-connected component of $\Psi(\Gamma g)^{-1}(V-A)$ is infinite then there exists a set $F_{0} \subset \mathbb{F}$ that is $S$-connected, infinite and $\Psi(\Gamma g)(f) \in V-A$ for all $f \in F_{0}$. The last condition implies that $\Gamma g \phi(f) K \notin T$ for all $f \in F_{0}$. Because $F_{0}$ is $S$-connected, the choice of $T$ implies that there is a component $H_{0}$ of $Q^{-1}\left(T^{c}\right)$ such that $g \phi\left(F_{0}\right) K \subset H_{0}$. Since $\phi\left(F_{0}\right)$ is infinite and discrete, there exist a point $l \in L(\phi(\mathbb{F})) \subset \partial \mathbb{H}^{n}$ in the closure of $\phi\left(F_{0}\right) K$. Then $g l$ is in the closure of $H_{0}$. Therefore, $g l$ is in the cusp set $C$. This proves the claim.

For $c \in C$, let $G_{c}=\left\{g \in G \mid g^{-1} c \in L(\phi(\mathbb{F}))\right\}$. Because $\phi(\mathbb{F})$ is a convex cocompact free group, $L(\phi(\mathbb{F}))$ has measure zero in $\partial \mathbb{H}^{n}$ (with respect to Lebesgue measure). Therefore, $G_{c}$ has Haar measure zero. Since $C$ is countable, $\bigcup_{c \in C} G_{c}$ has Haar 
measure zero. By the claim, the set of all $g \in G$ such that some $S$-connected component of $\Psi(\Gamma g)^{-1}(V-A)$ is infinite is contained in $\bigcup_{c \in C} G_{c}$. So it has measure zero. This proves the lemma.

As in Section 3.1, let $\delta>0$ be such that for all $g_{1}, g_{2} \in G$ with $d\left(g_{1}\right.$,id $)<\delta$ and $d\left(g_{2}\right.$, id $)<\delta$, if $s \in S$ then $d\left(g_{1} \phi(s) g_{2}, \phi(s)\right)<\epsilon$.

Let $\mu$ be the probability measure on $\Gamma \backslash G$ induced by Haar measure on $G$. Choose a Borel partition $V=\left\{v_{1}, v_{2}, \ldots\right\}$ of $\Gamma \backslash G$ into sets $v_{i}$ of diameter less than $\delta$ such that $\Gamma \in v_{1}, \mu\left(v_{1}\right)>0$ and $V$ satisfies the conclusion of the lemma above.

Let $\mathcal{G}$ be the graph with vertex set $V=\left\{v_{1}, v_{2}, \ldots\right\}$ and edges defined as follows. For each $v, w \in V$, if there exists elements $p \in v, q \in w$ and $s \in S$ such that $p \phi(s)=q$ then there is a directed edge in $\mathcal{G}$ from $v$ to $w$ labeled $s$. There are no other edges.

Let $X \subset V^{\mathbb{F}}$ be the graph subshift determined by $\mathcal{G}$. As in Section 3.2, for every $x \in X$, there is an $\epsilon$-perturbation $\phi_{x}: \mathbb{F} \rightarrow G$ of $\phi$.

Define $\Psi: \Gamma \backslash G \rightarrow X$ as in the lemma above. As in Section 3.3, this map commutes with the actions of $\mathbb{F}$. Therefore $\Psi_{*}(\mu)$ is a shift-invariant Borel probability measure on $X$. By the lemma above and Theorem 4.1, there exists a periodic treequence $z \in X$. Indeed, since $\mu\left(v_{1}\right)>0, \Psi_{*}(\mu)\left(\left\{x \in X \mid x\right.\right.$ (id) $\left.\left.=v_{1}\right\}\right)>0$. Thus, there exists a periodic treequence $z \in X$ such that $z(\mathrm{id})=v_{1}$. As in Section 3.3, $\phi_{z}$ is a $\epsilon$-perturbation of $\phi$ that is virtually a homomorphism into $\Gamma$. This proves Theorem 1.2.

\section{Asymptotic geometric properties}

The goal of this section is to prove Theorem 1.3. For the reader's convenience, the next subsection defines the terms used in the statement of the theorem.

\subsection{Definitions}

A nice reference for all the concepts below is Bridson and Haefliger [3].

Definition 2 Let $(X, d)$ be a metric space. A geodesic is a map $\gamma: I \rightarrow X$ where $I \subset \mathbb{R}$ is an interval and $d\left(\gamma(t), \gamma\left(t^{\prime}\right)\right)=\left|t-t^{\prime}\right|$ for all $t^{\prime}, t \in[a, b]$. It is a geodesic ray from $x \in X$ if, in addition, $I=[0, \infty)$ and $\gamma(0)=x$. A geodesic from $x$ to $y \in X$ is a geodesic of the form $\gamma:[a, b] \rightarrow X$ with $\gamma(a)=x$ and $\gamma(b)=y$. The image of $\gamma$ is a geodesic segment from $x$ to $y$ and is commonly denoted $[x, y]$ (although it depends on $\gamma$ and not just on $x$ and $y$ ). A geodesic triangle with vertices $x, y, z \in X$ is a union of three geodesic segments; one from $x$ to $y$, one from $y$ to $z$ and one 
from $z$ to $x .(X, d)$ is a geodesic space if for any two points $x, y \in X$ there exists a geodesic between them.

For $\delta \geq 0$, a metric space $(X, d)$ is $\delta$-hyperbolic if it is a geodesic space and for every geodesic triangle $\Delta \subset X$, each side of $\Delta$ is contained in the $\delta$-neighborhood of the union of the other two sides. If $(X, d)$ is $\delta$-hyperbolic for some $\delta \geq 0$, then we say it is Gromov-hyperbolic.

Definition 3 (Gromov boundary) Let $(X, d)$ be a proper metric space. Two geodesic rays $\gamma_{1}:[0, \infty) \rightarrow X, \gamma_{2}:[0, \infty) \rightarrow X$ are equivalent if there exists a $K \geq 0$ such that for any $t \geq 0,\left|\gamma_{1}(t)-\gamma_{2}(t)\right| \leq K$. The Gromov boundary of $X$, denoted $\partial X$, is the set of equivalence classes of geodesic rays.

If $\gamma:[0, \infty) \rightarrow X$ is a geodesic ray in the equivalence class $\xi \in \partial X$, then we say that $\gamma$ limits on $\xi$ and write $\gamma(\infty)=\xi$.

If $(X, d)$ is $\delta$-hyperbolic for some $\delta \geq 0$, then given two points $\xi_{1}, \xi_{2} \in \partial X$, there exists a geodesic $\gamma:(-\infty,+\infty) \rightarrow X$ such that the map $t \mapsto \gamma(-t)$ is a geodesic ray limiting on $\xi_{1}$ and the map $t \mapsto \gamma(t)$ is a geodesic ray limiting on $\xi_{2}$ [3, Chapter III.H, Lemma 3.2]. In this case, we say that $\gamma$ is a geodesic from $\xi_{1}$ to $\xi_{2}$ and write $\gamma(-\infty)=\xi_{1}, \gamma(+\infty)=\xi_{2}$.

Definition 4 A generalized ray is a geodesic $\gamma: I \rightarrow X$ where either $I=[0, R]$ for some $R>0$ or $I=[0, \infty)$. In the former case, define $\gamma(t)=\gamma(R)$ for all $t \in[R, \infty]$. We say that $\gamma$ is a generalized ray from $\gamma(0)$ to $\gamma(R)$.

Definition 5 Let $(X, d)$ be a proper Gromov-hyperbolic space with basepoint $p \in X$. We topologize $X \cup \partial X$ as follows. Say that a sequence $\left\{x_{i}\right\}_{i=1}^{\infty}$ converges to $x_{\infty}$ if and only if there exists generalized rays $\gamma_{i}$ from $p$ to $x_{i}$ such that every subsequence of $\left\{\gamma_{i}\right\}$ has a subsequence that converges uniformly on compact subsets to a geodesic from $p$ to $x_{\infty}$.

It is well-known that this defines a topology which is independent of $p$ and makes $X \cup \partial X$ a compact space in which $\partial X$ is closed (eg, [3, Chapter III.H, Proposition 3.7]).

Definition 6 For any subset $Y \subset X \cup \partial X$, let $L(Y)$ be the intersection of $\partial X$ with the closure of $Y$ in $X \cup \partial X$.

Definition 7 (Visual metric) Let $(X, d)$ be a proper Gromov-hyperbolic space with basepoint $p \in X$. A metric $d_{\partial}$ on $\partial X$ is called a visual metric with parameter $a>0$, 
if it induces the same topology on $\partial X$ as given above and there exists a constant $C>0$ such that for all $\xi_{1}, \xi_{2} \in \partial X$, if $\gamma$ is a geodesic from $\xi_{1}$ to $\xi_{2}$ then

$$
C^{-1} a^{-d(p, \gamma)} \leq d_{\partial}\left(\xi_{1}, \xi_{2}\right) \leq C a^{-d(p, \gamma)} .
$$

Here, $d(p, \gamma)=\inf _{t} d(p, \gamma(t))$.

Definition 8 A subset $Y \subset X$ is $\epsilon$-quasi-convex if any geodesic segment $[x, y]$ between points $x, y \in Y$ is contained in the $\epsilon$-neighborhood of $Y$. We say that $Y$ is quasi-convex if it is $\epsilon$-quasiconvex for some $\epsilon>0$.

Definition 9 An infinite group $\Gamma$ acting by isometries on a proper Gromov-hyperbolic space $(X, d)$ is called quasi-convex cocompact if the action is properly discontinuous, $\Gamma$ does not fix any point of $\partial X$, and for some $\Gamma$-invariant quasi-convex subset $A \subset X$, the quotient $A / \Gamma$ is compact.

\subsection{Quasi-isometries and quasi-geodesics}

In order to prove Theorem 1.3, we show (in the next subsection) that if $p \in X$ and $\epsilon>0$ is sufficiently small, then the map $h \mapsto \phi_{\epsilon}(h) p$ is a quasi-isometry of $H$ into $X$ (with respect to a fixed word metric on $H$ ). In this subsection, we introduce the necessary definitions and standard results needed to prove this.

Definition 10 Let $\left(X, d_{X}\right),\left(Y, d_{Y}\right)$ be metric spaces, $\lambda \geq 1, c \geq 0$. A map $\pi: X \rightarrow Y$ is a $(\lambda, c)$-quasi-isometric embedding if for all $x, y \in X$,

$$
\lambda^{-1} d_{X}(x, y)-c \leq d_{Y}(\pi(x), \pi(y)) \leq \lambda d_{X}(x, y)+c .
$$

We say that $\pi$ is a quasi-isometric embedding if it is a $(\lambda, c)$-quasi-isometric embedding for some constants $\lambda \geq 1, c \geq 0$.

Definition 11 For $\lambda \geq 1, c \geq 0$ a $(\lambda, c)$-quasi-geodesic in a metric space $(X, d)$ is a $(\lambda, c)$-quasi-isometric embedding $q: I \rightarrow X$ where $I$ is an interval on the real line (bounded or unbounded) or else the intersection of $\mathbb{Z}$ with such an interval. It is a quasi-geodesic ray if $I=[0, \infty)$ or $[0, \infty) \cap \mathbb{Z}$.

The theorem below is proven in [3, Chapter III.H, Theorem 1.7].

Theorem 6.1 (Stability of quasi-geodesics) For all $\delta \geq 0, \lambda \geq 1, c \geq 0$, there exists a constant $R=R(\delta, \lambda, c)$ with the following property.

Let $(X, d)$ denote a proper $\delta$-hyperbolic space. If $q: I \rightarrow X$ is a $(\lambda, c)$-quasi-geodesic in $X$ and $[x, y]$ is a geodesic segment joining the endpoints of $q$, then the Hausdorff distance between $[x, y]$ and the image of $q$ is less than $R$. 
Definition 12 Let $(X, d)$ be a metric space and $M \geq 0, \lambda \geq 1, c \geq 0$. A path $q: I \rightarrow X$ is said to be an $(M, \lambda, c)$-local-quasi-geodesic if for all $a, b \in I$ with $0 \leq b-a \leq M$, the restriction of $q$ to $[a, b]$ is a $(\lambda, c)$-quasi-geodesic.

Theorem 6.2 below states that any $(M, \lambda, c)$-local-quasi-geodesic in a $\delta$-hyperbolic space is a $\left(\lambda^{\prime}, c^{\prime}\right)$-quasi-geodesic for some $\left(\lambda^{\prime}, c^{\prime}\right)$ that depend only on $\delta, \lambda$ and $c$. This is stated in [9, Remark 7.2B] and proven in [5, Théorème 1.4].

Theorem 6.2 For any $\delta \geq 0, \lambda>1$ and $c \geq 0$, there exists $M_{1} \geq 0, K \geq 0, \lambda^{\prime}=\lambda^{\prime} \geq 1$ and $c^{\prime} \geq 0$ such that the following holds. Let $(X, d)$ be a $\delta$-hyperbolic space. Let $q: I \rightarrow X$ be an $(M, \lambda, c)$-local-quasi-geodesic for some $M \geq M_{1}$. Then $q$ is a $\left(\lambda^{\prime}, 2 K\right)$-quasi-geodesic.

Definition 13 Let $X, Y$ be metric spaces and $0 \leq M, \lambda \geq 1, c \geq 0$. A map $q$ : $Y \rightarrow X$ is said to be an $(M, \lambda, c)$-local-quasi-isometric embedding if for all $y \in Y, q$ restricted to the ball of radius $M$ centered at $y$ is a $(\lambda, c)$-quasi-isometric embedding.

Corollary 6.3 Let $M_{1}, \lambda^{\prime}, c^{\prime}$ be as in Theorem 6.2. Let $(X, d)$ be a $\delta$-hyperbolic space and $\left(Y, d_{Y}\right)$ a geodesic space. If $M \geq M_{1}$ and $q: Y \rightarrow X$ is an $(M, \lambda, c)-$ localquasi-isometric embedding then $q$ is a $\left(\lambda^{\prime}, c^{\prime}\right)$-quasi-isometric embedding.

Proof If $\gamma: I \rightarrow Y$ is a geodesic then $q \circ \gamma$ is an $(M, \lambda, c)$-local-quasi-geodesic. The theorem above implies that $q \circ \gamma$ is a $\left(\lambda^{\prime}, c^{\prime}\right)$-quasi-geodesic. Since this is true for all $\gamma, q$ is a $\left(\lambda^{\prime}, c^{\prime}\right)$-quasi-isometry.

\subsection{Perturbations and quasi-isometric embeddings}

In this subsection, we take the first step in proving Theorem 1.3 by showing that if $\epsilon>0$ is sufficiently small, then for some $p \in X$, the orbit map $h \mapsto \phi_{\epsilon}(h) p$ is a quasi-isometric embedding.

Definition 14 Let $H$ be an abstract group with finite symmetric generating set $S$. The Cayley graph $\mathcal{C}$ of $H$ induced by $S$ is the graph with vertex set $H$ and so that for every $h \in H$ and $s \in S$ there is an edge from $h$ to $h s$. Let each edge be isometric with the unit interval and let $d_{S}$ denote the resulting path metric. This makes $\mathcal{C}$ a geodesic space.

From now on, fix $H, S$ as above. Let $(X, d)$ be a $\delta$-hyperbolic space. We assume that $X$ contains more than one point. Since $X$ must contain a nontrivial geodesic segment, this implies that it is uncountable. Let $\phi: H \rightarrow \operatorname{Isom}(X)$ be an injective homomorphism onto a quasi-convex cocompact subgroup. Let $d_{\mathrm{Isom}(X)}$ be a left-invariant metric on Isom $(X)$ inducing the topology of uniform convergence on compact sets. 
Lemma 6.4 Let $p \in X$. Define $\pi_{p}: H \rightarrow X$ by $\pi_{p}(h)=\phi(h) p$. Then $\pi_{p}$ is a quasi-isometric embedding.

Proof Let $A \subset X$ be a $\phi(H)$-invariant quasi-convex subset such that $A / \phi(H)$ is compact. Since $X$ is a geodesic space, there exists a $C>0$ such that for every $C^{\prime}>C$, the $C^{\prime}$-neighborhood $N_{C^{\prime}}(A)$ of $A$ is path-connected. This neighborhood is also $\phi(H)$-invariant, quasi-convex and $N_{C^{\prime}}(A) / \phi(H)$ is compact. By quasi-convexity, there is some constant $C^{\prime \prime}>0$ so that any geodesic segment with endpoints in $A$ lies in the $C^{\prime \prime}$-neighborhood of $A$. So after replacing $A$ with a neighborhood of $A$ if necessary, we may assume that $A$ is path-connected and for some $a \in A$ all geodesic segments with endpoints in $\phi(H) a$ lie inside $A$.

Let $d_{A}$ be the metric on $A$ defined by $d_{A}(x, y)$ equals the infimum of lengths of paths in $A$ from $x$ to $y$. By the Švarc-Milnor lemma, the map $h \mapsto \phi(h) a$ is a $(\lambda, c)$-quasi-isometric embedding for some $\lambda \geq 1, c \geq 0$ with respect to the metric $d_{A}$. This lemma was discovered in the fifties by Efremovič [7] and Švarc [17] and rediscovered by Milnor [15, Lemma 2]. It is also proven in Bridson and Haefliger [3]. Since $d_{A}$ equals $d$ on $\phi(H) a$, this implies that $h \mapsto \phi(h) a$ is a $(\lambda, c)$-quasi-isometric embedding with respect to $d$ as well.

For any $h, g \in H$,

$$
\begin{aligned}
|d(\phi(h) p, \phi(g) p)-d(\phi(h) a, \phi(g) a)| & \leq d(\phi(h) p, \phi(h) a)+d(\phi(g) a, \phi(g) p) \\
& =2 d(a, p) .
\end{aligned}
$$

Since $\lambda^{-1} d_{S}(h, g)-c \leq d(\phi(h) a, \phi(g) a) \leq \lambda d_{S}(h, g)+c$, this implies

$$
\lambda^{-1} d_{S}(h, g)-c-2 d(a, p) \leq d(\phi(h) p, \phi(g) p) \leq \lambda d_{S}(h, g)+c+2 d(a, p) .
$$

Hence $h \mapsto \phi(h) p$ is a $(\lambda, c+2 d(p, a))$-quasi-isometric embedding.

Lemma 6.5 For any $N, \sigma>0$ and any $p \in X$, there exists an $\epsilon_{0}>0$ such that the following holds. If $0 \leq \epsilon \leq \epsilon_{0}$ and $\phi_{\epsilon}: H \rightarrow G$ is an $\epsilon$-perturbation of $\phi$ then for all $g, h \in H$ with $d_{S}(g, h) \leq N$,

$$
\left|d(\phi(g) p, \phi(h) p)-d\left(\phi_{\epsilon}(g) p, \phi_{\epsilon}(h) p\right)\right| \leq \sigma .
$$

Proof Let $\epsilon_{0}>0$ be such that if $m \leq N, s_{1}, \ldots, s_{m} \in S$ and $s_{1}^{\prime}, \ldots, s_{m}^{\prime} \in \operatorname{Isom}(X)$ are such that $d_{\operatorname{Isom}(X)}\left(s_{i}^{\prime}, \phi\left(s_{i}\right)\right) \leq \epsilon_{0}$ then

$$
\left|d\left(s_{1}^{\prime} \cdots s_{m}^{\prime} p, p\right)-d\left(\phi\left(s_{1}\right) \cdots \phi\left(s_{m}\right) p, p\right)\right| \leq \sigma .
$$

Let $0 \leq \epsilon \leq \epsilon_{0}$ and let $\phi_{\epsilon}$ be an $\epsilon$-perturbation of $\phi$. Let $g, h \in H$ with $d_{S}(g, h) \leq N$. So $g=h s_{1} s_{2} \cdots s_{m}$ for some $s_{i} \in S$ and $m \leq N$. Because $\phi_{\epsilon}$ is an $\epsilon$-perturbation of $\phi$, 
there exist elements $s_{i}^{\prime} \in G$ with $d_{\mathrm{Isom}(\mathrm{X})}\left(s_{i}^{\prime}, \phi\left(s_{i}\right)\right) \leq \epsilon$ and $\phi_{\epsilon}(g)=\phi_{\epsilon}(h) s_{1}^{\prime} \cdots s_{m}^{\prime}$. Thus

$$
\begin{aligned}
\mid d(\phi(g) p, \phi(h) p)-d & \left(\phi_{\epsilon}(g) p, \phi_{\epsilon}(h) p\right) \mid \\
& =\left|d\left(\phi\left(s_{1}\right) \cdots \phi\left(s_{m}\right) p, p\right)-d\left(s_{1}^{\prime} \cdots s_{m}^{\prime} p, p\right)\right| \leq \sigma .
\end{aligned}
$$

Proposition 6.6 Let $p \in X$. There exists an $\epsilon_{0}>0, \lambda^{\prime} \geq 1$ and $c^{\prime} \geq 0$ such that if $0 \leq \epsilon \leq \epsilon_{0}$ and $\phi_{\epsilon}: H \rightarrow \operatorname{Isom}(X)$ is any $\epsilon$-perturbation of $\phi$, then the map $h \mapsto \phi_{\epsilon}(h) p$ is a $\left(\lambda^{\prime}, c^{\prime}\right)$-quasi-isometric embedding of $H$ into $X$.

Proof By Lemma 6.4, the map $h \mapsto \phi(h) p$ is a $(\lambda, c)$-quasi-isometric embedding for some $\lambda \geq 1, c \geq 0$. Let $\sigma \geq 0$. Let $M \geq M_{1}$ where $M_{1}=M_{1}(\delta, \lambda+\sigma, c+\sigma+2 \lambda+2)$ is as defined in Corollary 6.3.

By the previous lemma, it follows that there exists an $\epsilon_{0}$ such that if $0 \leq \epsilon \leq \epsilon_{0}$ and $\phi_{\epsilon}: H \rightarrow \operatorname{Isom}(X)$ is an $\epsilon$-perturbation of $\phi$, then the map $h \mapsto \phi_{\epsilon}(h) p$ is an $(M, \lambda+\sigma, c+\sigma)$-local-quasi-isometric embedding. We can extend this map to the Cayley graph $\mathcal{C}$. For example, for each edge we could choose an endpoint and map the entire edge to the image of that endpoint. The resulting map is an $(M, \lambda+\sigma, c+\sigma+2 \lambda+2)$-local-quasi-isometric embedding. By Corollary 6.3, the map $h \mapsto \phi_{\epsilon}(h) p$ is a $\left(\lambda^{\prime}, c^{\prime}\right)$-quasi-isometry for some constants $\lambda^{\prime} \geq 1, c^{\prime} \geq 0$.

\subsection{Bi-Lipschitz maps}

In this section, we conclude that there exists $p \in X$ such that the map $\phi(h) p \mapsto \phi_{\epsilon}(h) p$ is bi-Lipschitz with constant that tends to 1 as $\epsilon$ tends to 0 . This result is the key ingredient to proving Theorem 1.3. We need the next two lemmas.

Lemma 6.7 There exists a point $p \in X$ such that $p$ is not a fixed point of any nontrivial element of $\phi(H)$. For any such point, there exists a $\rho>0$ such that if $g, h \in H$ and $g \neq h$ then $d(\phi(g) p, \phi(h) p) \geq \rho$.

Proof It is well-known that any nonidentity isometry of a Gromov-hyperbolic space fixes at most 1 point. Since $H$ is countable and $X$ is uncountable, there exists a point $p$ that is not fixed by any nontrivial element of $\phi(H)$.

By Lemma 6.4, there exists $\lambda \geq 1$ and $c \geq 0$ such that the map $f \mapsto \phi(f) p$ is a $(\lambda, c)$-quasi-isometric embedding of $H$ into $X$. Let $N$ be an integer such that $\lambda^{-1} N-c \geq 1$. Let

$$
\rho_{0}=\min \left\{d(p, \phi(g) p) \mid d_{S}(g, \mathrm{id}) \leq N, g \neq \mathrm{id}\right\} .
$$


Because $p$ is not fixed by any nontrivial element of $\phi(H), \rho_{0}>0$. Let $g, h \in H$ with $g \neq h$. If $d_{S}(g, h) \leq N$ then $d(\phi(g) p, \phi(h) p)=d\left(\phi\left(h^{-1} g\right) p, p\right) \geq \rho_{0}$. If $d_{S}(g, h)>$ $N$ then, since the map $f \mapsto \phi(f) p$ is a $(\lambda, c)$-quasi-isometry, $d(\phi(g) p, \phi(h) p) \geq$ $\lambda^{-1} N-c \geq 1$. Set $\rho=\min \left(1, \rho_{0}\right)$ to finish the lemma.

Lemma 6.8 Let $R=R(\delta, \lambda, c)$ be as in Theorem 6.1. If $Y$ is a geodesic space and $\psi: Y \rightarrow X$ a $(\lambda, c)$-quasi-isometric embedding then $\psi(Y)$ is $R$-quasi-convex.

Proof Let $x, y \in Y$ and let $[\psi(x), \psi(y)]$ be a geodesic in $X$ from $\psi(x)$ to $\psi(y)$. Let $\gamma: I \rightarrow Y$ be a geodesic segment from $x$ to $y$. Then $\psi \circ \gamma$ is a $(\lambda, c)$-quasi-geodesic from $\psi(x)$ to $\psi(y)$ for some $\lambda \geq 1, c \geq 0$ that depend only on $\psi$ and not on $x$ and $y$. By Theorem 6.1, $[\psi(x), \psi(y)]$ is contained in the $R$-neighborhood of $\psi \circ \gamma(I)$. Since the latter is contained in $\psi(Y),[\psi(x), \psi(y)]$ is in the $R$-neighborhood of $\psi(Y)$. Since $\gamma$ is arbitrary, this implies $\psi(Y)$ is $R$-quasi-convex.

Proposition 6.9 Let $p \in X$ be such that no nontrivial element of $\phi(H)$ fixes $p$. Let $\kappa>1$. Then there exists an $\epsilon_{3}>0$ such that if $0 \leq \epsilon \leq \epsilon_{3}$ and $\phi_{\epsilon}: H \rightarrow \operatorname{Isom}(X)$ is an $\epsilon$-perturbation of $\phi$, then for all $g, h \in H$,

$$
\kappa^{-1} d(\phi(h) p, \phi(g) p) \leq d\left(\phi_{\epsilon}(h) p, \phi_{\epsilon}(g) p\right) \leq \kappa d(\phi(h) p, \phi(g) p) .
$$

Proof By Proposition 6.6, there exists an $\epsilon_{0}>0, \lambda \geq 1, c \geq 0$ such that if $0 \leq \epsilon \leq \epsilon_{0}$ then the map $h \mapsto \phi_{\epsilon}(h) p$ is a $(\lambda, c)$-quasi-isometric embedding. By the previous lemma, there exists a $C>0$ such that $\phi(H) p$ and $\phi_{\epsilon}(H) p$ are $C$-quasi-convex (for any $\epsilon \leq \epsilon_{0}$ ). By Lemma 6.7 and Lemma 6.5 there exists an $\epsilon_{1}>0$ and $\rho>0$ such that if $0 \leq \epsilon \leq \epsilon_{1}$ then for all $g, h \in H$ with $g \neq h$,

$$
d(\phi(g) p, \phi(h) p) \geq \rho \quad \text { and } \quad d\left(\phi_{\epsilon}(g) p, \phi_{\epsilon}(h) p\right) \geq \rho .
$$

Let $\sigma>0$ be small enough so that $1+\sigma / \rho<\kappa$. Let $N>0$ be an integer such that

$$
1<1+\frac{2(\sigma+2 C)}{\lambda^{-1} N-c}<\kappa
$$

By Lemma 6.5, there exists $\epsilon_{2}>0$ such that if $g, h \in H$ are such that $d_{S}(g, h) \leq N$ and $0 \leq \epsilon \leq \epsilon_{2}$, then

$$
\left|d\left(\phi_{\epsilon}(g) p, \phi_{\epsilon}(h) p\right)-d(\phi(g) p, \phi(h) p)\right| \leq \sigma .
$$


We now assume that $0 \leq \epsilon \leq \epsilon_{3}:=\min \left(\epsilon_{0}, \epsilon_{1}, \epsilon_{2}\right)$. Let $g, h \in H$. Suppose that $d_{S}(g, h) \leq N$. Then by the definition of $\epsilon_{2}$ and the choice of $\sigma$,

$$
\begin{aligned}
\kappa^{-1} d(\phi(h) p, \phi(g) p) & \leq \kappa^{-1}\left(d\left(\phi_{\epsilon}(h) p, \phi_{\epsilon}(g) p\right)+\sigma\right) \\
& \leq \kappa^{-1}\left(1+\frac{\sigma}{\rho}\right) d\left(\phi_{\epsilon}(h) p, \phi_{\epsilon}(g) p\right) \\
& \leq d\left(\phi_{\epsilon}(h) p, \phi_{\epsilon}(g) p\right) \\
& \leq d(\phi(h) p, \phi(g) p)+\sigma \\
& \leq\left(1+\frac{\sigma}{\rho}\right) d(\phi(h) p, \phi(g) p) \\
& \leq \kappa d(\phi(h) p, \phi(g) p) .
\end{aligned}
$$

This proves the theorem in the case $d_{S}(g, h) \leq N$. So suppose $d_{S}(g, h)>N$. Then there exists elements $g_{0}, g_{1}, \ldots, g_{k} \in H$ with $k \geq 2$ such that

- $g_{0}=g, g_{k}=h$,

- $\sum_{i=0}^{k-1} d_{S}\left(g_{i}, g_{i+1}\right)=d_{S}(g, h)$,

- $d_{S}\left(g_{0}, g_{1}\right) \leq N$ and

- $d_{S}\left(g_{i}, g_{i+1}\right)=N$ for $1 \leq i \leq k-1$.

Let $x_{i}=\phi\left(g_{i}\right) p$ and $x_{i}^{\prime}=\phi_{\epsilon}\left(g_{i}\right) p$. By definition of $\epsilon_{2}$, for any $i$,

$$
\left|d\left(x_{i}, x_{i+1}\right)-d\left(x_{i}^{\prime}, x_{i+1}^{\prime}\right)\right| \leq \sigma .
$$

Since $\phi(H) p$ is $C$-quasi-convex,

$$
-2 C k+\sum_{i=0}^{k-1} d\left(x_{i}, x_{i+1}\right)=\sum_{i=0}^{k-1}\left(d\left(x_{i}, x_{i+1}\right)-2 C\right) \leq d\left(x_{0}, x_{k}\right),
$$

Since $\phi$ is a $(\lambda, c)$-quasi-isometry,

Thus,

$$
\begin{aligned}
d\left(x_{0}, x_{k}\right) & \geq \lambda^{-1} d_{S}(g, h)-c \geq \lambda^{-1}(k-1) N-c . \\
d\left(x_{0}^{\prime}, x_{k}^{\prime}\right) & \leq \sum_{i=0}^{k-1} d\left(x_{i}^{\prime}, x_{i+1}^{\prime}\right) \leq \sum_{i=0}^{k-1} d\left(x_{i}, x_{i+1}\right)+\sigma \\
& \leq d\left(x_{0}, x_{k}\right)+\sigma k+2 C k=d\left(x_{0}, x_{k}\right)+(\sigma+2 C) k \\
& \leq d\left(x_{0}, x_{k}\right)+\frac{(\sigma+2 C) k d\left(x_{0}, x_{k}\right)}{\lambda^{-1}(k-1) N-c} .
\end{aligned}
$$


Observe that

$$
\frac{(\sigma+2 C) k}{\lambda^{-1}(k-1) N-c}=\frac{(\sigma+2 C)\left(\frac{k}{k-1}\right)}{\lambda^{-1} N-\frac{c}{k-1}} \leq \frac{2(\sigma+2 C)}{\lambda^{-1} N-c} \leq \kappa-1 .
$$

Thus we have shown that $d\left(x_{0}^{\prime}, x_{k}^{\prime}\right) \leq \kappa d\left(x_{0}, x_{k}\right)$. Equivalently,

$$
d\left(\phi_{\epsilon}(h) p, \phi_{\epsilon}(g) p\right) \leq \kappa d(\phi(h) p, \phi(g) p) .
$$

Observe that all of the above inequalities and equations remain true if the roles of $\phi$ and $\phi_{\epsilon}$ are switched. Therefore,

$$
d(\phi(h) p, \phi(g) p) \leq \kappa d\left(\phi_{\epsilon}(h) p, \phi_{\epsilon}(g) p\right) .
$$

This implies the theorem.

\subsection{Proof of Theorem 1.3}

We will prove each item of Theorem 1.3 separately.

Proposition 6.10 There exists an $\epsilon_{0}>0$ such that if $0 \leq \epsilon \leq \epsilon_{0}$ and $\phi_{\epsilon}: H \rightarrow G$ is an $\epsilon$-perturbation of $\phi$ then $\phi_{\epsilon}$ is $1-1$.

Proof Let $p \in X$ and $\rho>0$ be as in Lemma 6.7. Let $\kappa>1$. By Proposition 6.9 there exists an $\epsilon_{0}>0$ such that if $0 \leq \epsilon \leq \epsilon_{0}$ then for all $g, h \in H$ with $g \neq h$,

$$
0<\kappa^{-1} \rho \leq \kappa^{-1} d(\phi(h) p, \phi(g) p) \leq d\left(\phi_{\epsilon}(h) p, \phi_{\epsilon}(g) p\right) .
$$

Fix a visual metric $d_{\partial}$ on $\partial X$. The next lemma is well-known. For example, it is an immediate consequence of Lemma 3.6 in [3, Chapter III.H].

Lemma 6.11 Let $p \in X, W>0$ and $C>0$. Then there exists a $N \geq 0$ such that if $q_{1}:[0, \infty) \rightarrow X$ and $q_{2}:[0, \infty) \rightarrow X$ are geodesic rays with $q_{1}(0)=q_{2}(0)=p$ and $d\left(q_{1}(N), q_{2}(N)\right)<W$ then $d_{\partial}\left(q_{1}(\infty), q_{2}(\infty)\right)<C$.

Because the map $h \mapsto \phi(h) p$ is a quasi-isometric embedding into a $\delta$-hyperbolic space, it follows that the Cayley graph of $H$, denoted $\mathcal{C}$, is Gromov-hyperbolic. Let $\partial H=\partial \mathcal{C}$.

Proposition 6.12 For all $C>0$ and $p \in X$, there exists an $\epsilon_{0}>0$ such that if $0 \leq \epsilon \leq \epsilon_{0}$ and $\phi_{\epsilon}: H \rightarrow G$ is an $\epsilon$-perturbation of $\phi$ such that $\phi_{\epsilon}(\mathrm{id})=\mathrm{id}$ then

$$
d_{\text {Haus }}\left(L(\phi(H) p), L\left(\phi_{\epsilon}(H) p\right)\right)<C .
$$


Proof By Proposition 6.6, there exists $\lambda^{\prime} \geq 1, c^{\prime} \geq 0, \epsilon_{2}>0$ such that if $0 \leq \epsilon \leq \epsilon_{2}$ then the map $h \mapsto \phi_{\epsilon}(h) p$ is a $\left(\lambda^{\prime}, c^{\prime}\right)$-quasi-isometry.

Let $\sigma>0$ and let $W:=4 R+2 \sigma$ where $R=R\left(\delta, \lambda^{\prime}, c^{\prime}\right)$ is as in Theorem 6.1. Let $N$ be as in the previous lemma.

By the proof of Lemma 6.5 and the assumption $\phi_{\epsilon}(\mathrm{id})=\mathrm{id}$, there exists an $\epsilon_{1}>0$ such that if $0 \leq \epsilon \leq \epsilon_{1}$ then for all $h \in H$ with $d_{S}(h$,id $) \leq \lambda^{\prime}(N+R)+\lambda^{\prime} c^{\prime}$, $d\left(\phi_{\epsilon}(h) p, \phi(h) p\right) \leq \sigma$.

Now let $\epsilon_{0}=\min \left(\epsilon_{1}, \epsilon_{2}\right)$. Let $0 \leq \epsilon \leq \epsilon_{0}$. Let $\phi_{\epsilon}: H \rightarrow \operatorname{Isom}(X)$ be any $\epsilon-$ perturbation of $\phi$. Let $\pi_{\epsilon}: H \rightarrow X$ be the map $\pi_{\epsilon}(h)=\phi_{\epsilon}(h) p$. Extend $\phi_{\epsilon}$ to all of the Cayley graph $\mathcal{C}$ by choosing, for each edge in $\mathcal{C}$, one of its endpoints and mapping the entire edge to the image of its endpoint. The resulting map is still a quasi-isometric embedding. By [3, Chapter III.H, Theorem 3.9], $\pi_{\epsilon}$ has a unique continuous extension $\pi_{\epsilon}: \mathcal{C} \cup \partial \mathcal{C} \rightarrow X \cup \partial X$ that restricts to a topological embedding of $\partial \mathcal{C}$ into $\partial X$ with image equal to $L\left(\phi_{\epsilon}(H) p\right)$.

Similarly, let $\pi_{0}: H \rightarrow X$ be the map $\pi_{0}(h)=\phi(h) p$. Extend it to a map $\pi_{0}: \mathcal{C} \cup \partial \mathcal{C} \rightarrow$ $X \cup \partial X$ in a similar manner.

Let $\xi \in \partial H$. Let $q:[0, \infty) \rightarrow \mathcal{C}$ be a geodesic ray with $q(0)=$ id and $q(\infty)=\xi$. By definition $\pi_{\epsilon} \circ q$ is a $\left(\lambda^{\prime}, c^{\prime}\right)$-quasi-geodesic. Let $\zeta_{\epsilon}=\pi_{\epsilon}(\xi)$. Let $\gamma_{\epsilon}:[0, \infty) \rightarrow X$ be a geodesic ray with $\gamma_{\epsilon}(0)=p$ and $\gamma_{\epsilon}(\infty)=\zeta_{\epsilon}$. Similarly, let $\gamma_{0}:[0, \infty) \rightarrow X$ be a geodesic ray with $\gamma_{0}(0)=p$ and $\gamma_{0}(\infty)=\zeta_{0}$.

By Theorem 6.1, the Hausdorff distance between $\pi_{\epsilon} \circ q([0, \infty) \cap \mathbb{Z})$ and $\gamma_{\epsilon}([0, \infty))$ is at most $R$. So there exists an $h \in H$ with $d\left(\gamma_{\epsilon}(N), \pi_{\epsilon}(h)\right) \leq R$. Since $\pi_{\epsilon}$ is a $\left(\lambda^{\prime}, c^{\prime}\right)$-quasi-isometry,

$$
d_{S}(h, \mathrm{id}) \leq \lambda^{\prime} d\left(\pi_{\epsilon}(h), p\right)+\lambda^{\prime} c^{\prime} \leq \lambda^{\prime}(N+R)+\lambda^{\prime} c^{\prime} .
$$

By definition of $\epsilon_{1}, d\left(\pi_{\epsilon}(h), \pi_{0}(h)\right) \leq \sigma$. By Theorem 6.1, there exists a $t \geq 0$ with $d\left(\pi_{0}(h), \gamma_{0}(t)\right) \leq R$. Observe that

$$
\begin{aligned}
|t-N| & =\left|d\left(\gamma_{0}(t), p\right)-d\left(\gamma_{\epsilon}(N), p\right)\right| \leq d\left(\gamma_{0}(t), \gamma_{\epsilon}(N)\right) \\
& \leq d\left(\gamma_{0}(t), \pi_{0}(h)\right)+d\left(\pi_{0}(h), \pi_{\epsilon}(h)\right)+d\left(\pi_{\epsilon}(h), \gamma_{\epsilon}(N)\right) \leq 2 R+\sigma .
\end{aligned}
$$

This implies

$$
\begin{aligned}
d\left(\gamma_{0}(N), \gamma_{\epsilon}(N)\right) & \leq\left|d\left(\gamma_{0}(N), p\right)-d\left(\gamma_{0}(t), p\right)\right|+\left|d\left(\gamma_{0}(t), p\right)-d\left(\gamma_{\epsilon}(N), p\right)\right| \\
& \leq 4 R+2 \sigma .
\end{aligned}
$$

By the choice of $N$, this implies that $d_{\partial}\left(\pi_{\epsilon}(\xi), \pi_{0}(\xi)\right) \leq C$. Since this is true for all $\xi \in \partial H$, it follows that $d_{\text {Haus }}\left(\pi_{\epsilon}(\partial H), \pi_{0}(\partial H)\right) \leq C$ as claimed. 
To prove the last part of Theorem 1.3, we rely on a well-known generalization of Patterson-Sullivan theory to word hyperbolic groups due to Coornaert. This is explained next.

Definition 15 Let $\Gamma \subset \operatorname{Isom}(X)$ be a discrete subset, $p, q \in X$ and $s>0$. Then the Poincaré series of $\Gamma$ with respect to the visual parameter $a>0$ is defined by

$$
g_{s}(p, q)=\sum_{\gamma \in \Gamma} a^{-s d(p, \gamma q)} .
$$

A short calculation shows that if $g_{s}(p, q)$ is finite for some pair $(p, q)$ then it is finite for all such pairs. So let $\delta_{a}(\Gamma)=\inf \left\{s: g_{s}(p, q)<\infty\right\}$ be the exponent of convergence of $\Gamma$ with respect to the parameter $a$. In [4] it was proven that if $\Gamma$ is a quasi-convex cocompact subgroup then $\delta_{a}(\Gamma)=\mathcal{H} \mathcal{D}(L(\Gamma))$, the Hausdorff-dimension of the limit set of $\Gamma$ with respect to a visual metric $d_{\partial}$ with parameter $a$.

In order to apply Coornaert's result, we need the next lemma.

Lemma 6.13 There exists an $\epsilon_{0}>0$ such that if $0 \leq \epsilon \leq \epsilon_{0}, \phi_{\epsilon}: H \rightarrow \operatorname{Isom}(X)$ is an $\epsilon$-perturbation of $\phi$ and $\phi_{\epsilon}$ restricted to $H^{\prime}$ is a homomorphism (for some $H^{\prime}<H$ with finite index) then $\phi_{\epsilon}\left(H^{\prime}\right)$ is quasi-convex cocompact.

Proof Let $p \in X$. By Proposition 6.6, there exists $\epsilon_{0}>0$ such that if $0 \leq \epsilon \leq \epsilon_{0}$ then the map $h \mapsto \phi_{\epsilon}(h) p$ is a quasi-isometry. By Lemma 6.8, if $A=\left\{\phi_{\epsilon}(h) p \mid h \in H^{\prime}\right\}$, then $A$ is quasi-convex. Of course, it is $\phi_{\epsilon}\left(H^{\prime}\right)$-invariant. Since $A / \phi_{\epsilon}\left(H^{\prime}\right)$ is a single point, it is compact.

Proposition 6.14 Let $C>0$. There exists an $\epsilon_{0}>0$ such that if $0 \leq \epsilon \leq \epsilon_{0}$ and $\phi_{\epsilon}: H \rightarrow \operatorname{Isom}(X)$ is an $\epsilon$-perturbation of $\phi$ that is virtually a homomorphism then $\left|\mathcal{H D}\left(L\left(\phi_{\epsilon}\right)\right)-\mathcal{H D}(L(\phi))\right| \leq C$.

Proof It follows from Proposition 6.9 that there exists an $\epsilon_{0}>0$ such that if $0 \leq \epsilon \leq \epsilon_{0}$ and $\phi_{\epsilon}: H \rightarrow \operatorname{Isom}(X)$ is an $\epsilon$-perturbation of $\phi$ then $\left|\delta(\phi(H))-\delta\left(\phi_{\epsilon}(H)\right)\right|<C$.

Assume $\phi_{\epsilon}$ is virtually a homomorphism. So there exists a finite-index subgroup $H^{\prime}<H$ such that $\phi_{\epsilon}$ restricted to $H^{\prime}$ is a homomorphism. By the previous lemma and Coornaert's result (mentioned in Definition 15), $\delta_{a}\left(\phi_{\epsilon}\left(H^{\prime}\right)\right)=\mathcal{H D}\left(L\left(\phi_{\epsilon}\left(H^{\prime}\right) p\right)\right)$. So it suffices to show that $\delta_{a}\left(\phi_{\epsilon}\left(H^{\prime}\right)\right)=\delta_{a}\left(\phi_{\epsilon}(H)\right)$ and $L\left(\phi_{\epsilon}\left(H^{\prime}\right) p\right)=L\left(\phi_{\epsilon}(H) p\right)$. These are easy exercises left to the reader.

Theorem 1.3 now follows from Propositions 6.10, 6.12 and 6.14. 


\section{Surface groups and aperiodic tilings}

Because of the surface subgroup conjecture, we would like to generalize Theorem 1.1 to allow $F$ to be a surface group. The only step in the proof which requires $F$ to be a free group is in Theorem 2.1: showing the existence of a periodic point in a certain graph subshift over $F$. This points to a general problem: find conditions on graph subshifts over a surface group that guarantee the existence of a periodic point. We will consider a continuous version of this problem; replacing the surface group with $\mathrm{PSL}_{2}(\mathbb{R})=\operatorname{Isom}^{+}\left(\mathbb{H}^{2}\right)$ and show, by an explicit counterexample, that the existence of an invariant Borel probability measure is not sufficient. However, up to minor variations, this is the only known counterexample. We make a precise conjecture to the effect that this counterexample is unique and show that it implies the surface subgroup conjecture.

To begin, let us define tiling spaces, which are the continuous analog of graph subshifts.

Definition 16 A tile is a curvilinear polygon in $\mathbb{H}^{2}$. We think of it as a compact subset $\mathbb{H}^{2}$ (equal to the closure of its interior) and also as a finite CW-complex isomorphic to a polygon. If $P=\left\{\tau_{1}, \tau_{2}, \ldots\right\}$ is a set of tiles, then a tiling by $P$ is a collection $T$ of congruent copies of the tiles in $P$ such that

- (covering) the union of all tiles in $T$ equals the whole plane and

- (edge-to-edge) for any distinct pair $\tau_{1}, \tau_{2}$ of tiles in $T$ the intersection of $\tau_{1}$ with $\tau_{2}$ is either empty, a vertex of both, or an edge of both.

Let $\mathcal{T}(P)$ be the set of all tilings by $P$. It has the following topology. If $\left\{T_{i}\right\}$ is a sequence of tilings then $T_{i}$ converges to $T_{\infty}$ if for every tile $\tau \in T_{\infty}$, there exist tiles $\tau_{i} \in T_{i}$ with $\tau_{i}$ converging to $\tau$ in the Hausdorff topology on closed subsets of the plane.

Isom $^{+}\left(\mathbb{H}^{2}\right)$ acts on $\mathcal{T}(P)$ in the obvious way: for all $g \in \operatorname{Isom}^{+}\left(\mathbb{H}^{2}\right), g T=$ $\{g \tau \mid \tau \in T\}$. The symmetry group of $T$ is the group of all isometries that fix $T$. $T$ is periodic if its symmetry group is cofinite. If $P$ is finite, then the symmetry group of $T$ is necessarily discrete.

If the set of tiles $P$ is such that $\mathcal{T}(P)$ is nonempty and every tiling $T \in \mathcal{T}(P)$ is nonperiodic then $P$ is said to be aperiodic.

Here is a brief history of aperiodic tilings of the hyperbolic plane. The first aperiodic tile set $P$ is described by Penrose [16]. It has only one tile which is nonconvex. More examples were discovered by Margulis and Mozes [12]: indeed they discovered a set $P$ consisting of a single tile, a triangle, that admits only nonperiodic tilings. In 
Goodman-Strauss [8], an example is provided of a finite set $P$ of tiles such that each tiling $T \in \mathcal{T}(P)$ has no nontrivial symmetries. Dranishnikov and Schroeder [6] gave a new construction of aperiodic tile sets. The first example of an aperiodic tile set $P$ such that $\mathcal{T}(P)$ admits an invariant Borel probability measure appears in Bowen, Holton, Radin and Sadun [2]. It is sketched below. Except for minor variations it is the only example known.

Theorem 7.1 There exists an aperiodic set of tiles $P=\{\tau, \sigma\}$ such that $\mathcal{T}(P)$ is nonempty and there is a Isom ${ }^{+}\left(\mathbb{H}^{2}\right)$-invariant Borel probability measure on $\mathcal{T}(P)$.

Proof sketch This is only a sketch. For details, see Bowen et al [2]. The starting point is a slight modification of an aperiodic tile set described in [16]. Identify $\mathbb{H}^{2}$ with the upperhalf plane model. So $\mathbb{H}^{2}=\{x+i y \in \mathbb{C} \mid y>0\}$ with the metric $d s^{2}=\left(d x^{2}+d y^{2}\right) / y^{2}$. Euclidean similarities that preserve $\mathbb{H}^{2}$ are isometries of the hyperbolic metric.

For $w>0$, let $\sigma=\sigma_{w}$ be the Euclidean rectangle with vertices $i, i+w, 2 i, 2 i+w$. As a CW-complex, it is to be regarded as a pentagon where the extra vertex is at $i+w / 2$.

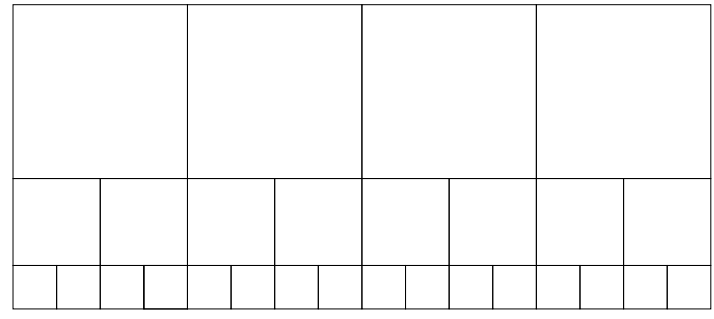

Figure 1: Part of a tiling by $\{\sigma\}$ in the upper-half plane model

Let $t, s$ be the isometries of $\mathbb{H}^{2}$ given by $t(z)=z+w$ and $s(z)=2 z$. Then $T=$ $\left\{s^{n} t^{m} \sigma_{w} \mid n, m \in \mathbb{Z}\right\}$ is a tiling by $P$. See Figure 1 . Thus $\mathcal{T}(\{\sigma\})$ is nonempty.

However, it can be shown that $\mathcal{T}(\{\sigma\})$ does not admit any $\operatorname{PSL}_{2}(\mathbb{R})$-invariant Borel probability measures. This is because there is a $\operatorname{PSL}_{2}(\mathbb{R})$-equivariant map from $\mathcal{T}(\{\sigma\})$ onto $\partial \mathbb{H}^{2}$ defined by $T \mapsto p$ where $p$ is the unique point which is contained in the geodesic extension of every "vertical" edge of tiles in $T$. If there is a $\mathrm{PSL}_{2}(\mathbb{R})$-invariant Borel probability measure on $\mathcal{T}(\{\sigma\})$ then it pushes forward to a $\mathrm{PSL}_{2}(\mathbb{R})$-invariant measures on $\partial \mathbb{H}^{2}$. But, an easy exercise shows that there are no $\mathrm{PSL}_{2}(\mathbb{R})$-invariant Borel probability measures on $\partial \mathbb{H}^{2}$. 
To get an invariant measure we will need another tile. Consider the standard horoball packing as shown in Figure 2 in the Poincaré model. It is invariant under $\operatorname{PSL}_{2}(\mathbb{Z})<$ $\mathrm{PSL}_{2}(\mathbb{R})$. Let $\tau$ be one of the curvilinear triangles in the complement of the horoballs. Let $w$ be the length of one of its edges.

Let $P=\left\{\tau, \sigma_{w}\right\}$. $P$ tiles in the following way. Consider the standard horoball packing. Tile each horoball with copies of $\sigma_{w}$. Use the triangle $\tau$ to tile the complement of the horoballs. See Figure 2. In [2] it was shown that $P$ is aperiodic and there are uncountably many ergodic $\mathrm{PSL}_{2}(\mathbb{R})$-invariant Borel probability measures on $\mathcal{T}(P)$. Here we only sketch aperiodicity and existence of a Borel probability measure.
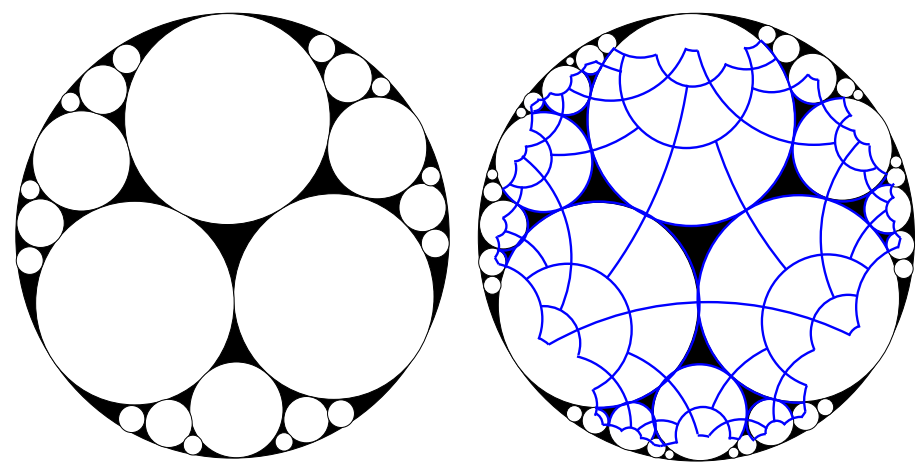

Figure 2: Left: Part of the horoball packing invariant under $\operatorname{PSL}_{2}(\mathbb{Z})$. Right: Part of a tiling by $\left\{\tau, \sigma_{w}\right\}$.

Let $T \in \mathcal{T}(P)$. Note that $T$ contains a copy of $\sigma_{w}$ since it is not possible to tile with copies of the triangle $\tau$ alone. Let $\sigma^{\prime}$ be such a copy. Note that only another copy of $\sigma_{w}$ is allowed to be next to $\sigma^{\prime}$ on either its left or its right edges. Therefore, $T$ contains a copy of the set $\left\{t^{i} \sigma_{w} \mid i \in \mathbb{Z}\right\}$ where $t$ is the isometry $t(z)=z+w$. So if $e$ is the "top" edge of $\sigma^{\prime}$ then the set $h=\left\{t^{i} e \mid i \in \mathbb{Z}\right\}$ is a horocycle. So there is a horocycle contained in the edges of $T$.

If $\Gamma$ is the symmetry group of $T$ then the quotient space $\mathbb{H}^{2} / \Gamma$ admits a tiling by $\{\sigma, \tau\}$ (the quotient tiling). So the horocycle $h$ must descend to a closed horocycle on $\mathbb{H}^{2} / \Gamma$. Thus $\mathbb{H}^{2} / \Gamma$ is noncompact. But any tile placed deep enough in a cusp necessarily has self-intersections. So $\mathbb{H}^{2} / \Gamma$ has no cusps. So it cannot have finite volume. This contradiction implies $T$ is nonperiodic. Since $T$ is arbitrary, $P$ is aperiodic.

To produce a $\mathrm{PSL}_{2}(\mathbb{R})$-invariant Borel probability measure on $\mathcal{T}(P)$, consider the standard horoball packing as in Figure 2. Use the triangle $\tau$ to tile the complement of the union of horoballs. For $N>0$, tile the first $N$ "rows" of each horoball with 
copies of $\sigma_{w}$. This can be done in such a way that the resulting partial tiling has symmetry group $H_{N}$ with finite index in $\operatorname{PSL}_{2}(\mathbb{Z})$. Hence $H_{N}$ is a lattice. So there is an invariant probability measure $\mu_{N}$ supported on the translates of this partial tiling.

The set of partial tilings of $\mathbb{H}^{2}$ by $P$ is topologized in a manner analogous to how $\mathcal{T}(P)$ is topologized. With this topology, it is compact and metrizable. So the BanachAlaoglu theorem implies the existence of a weak* limit point of the sequence $\left\{\mu_{N}\right\}$. Let $\mu$ be such a point. Given any fixed point $p \in \mathbb{H}^{2}$, the $\mu_{N}$-probability that $p$ is contained in a tile tends to 1 as $N \rightarrow \infty$. Hence $\mu$ is supported on full tilings (as opposed to partial tilings). Since each $\mu_{N}$ is $\mathrm{PSL}_{2}(\mathbb{R})$-invariant, $\mu$ is also $\mathrm{PSL}_{2}(\mathbb{R})-$ invariant.

Recall that the support of a measure $\mu$ on a topological space $X$ is the complement of the largest open subset $O$ with $\mu(O)=0$. It is denoted here by $\operatorname{support}(\mu)$. The action of a group $G$ on $X$ is minimal if every orbit $G x$ is dense in $X$.

In the example provided above, $\mathrm{PSL}_{2}(\mathbb{R})$ does not act minimally on the support of any invariant probability measure $\mu$ on $\mathcal{T}(P)$. Indeed, if $T$ is any tiling by $P$ then there exists a tiling $T^{\prime}$ in the closure of the $\operatorname{PSL}_{2}(\mathbb{R})$-orbit of $T$ that is a tiling by $\{\sigma\}$ alone. In fact, $\mathcal{T}(\{\sigma\})$ is contained in the closure of the $\mathrm{PSL}_{2}(\mathbb{R})$-orbit of $T$. $\mathrm{PSL}_{2}(\mathbb{R})$ acts minimally on $\mathcal{T}(\{\sigma\}) \subset \mathcal{T}(P)$, but no $\mathrm{PSL}_{2}(\mathbb{R})$-invariant Borel probability measure has support in $\mathcal{T}(\{\sigma\})$. I conjecture that this phenomenon holds for every finite aperiodic tile set:

Conjecture 1 Let $Q$ be an aperiodic finite set of tiles of $\mathbb{H}^{2}$. Suppose there exists a $\mathrm{PSL}_{2}(\mathbb{R})$-invariant Borel probability measure $\mu$ on $\mathcal{T}(Q)$. Then $\mathrm{PSL}_{2}(\mathbb{R})$ does not act minimally on the support of $\mu$.

Recall that a surface group is the fundamental group of a closed surface of genus at least 2. Here is a discrete form of the same conjecture:

Conjecture 2 Let $X \subset V^{\Sigma}$ be the graph subshift defined by a finite graph $\mathcal{G}$ where $\Sigma$ is a surface group. If there exists a shift-invariant Borel probability measure $\mu$ on $X$ such that $\Sigma$ acts minimally on the support of $\mu$ then there exists a periodic point $x \in X$ (ie, the stabilizer of $x$ has finite index in $\Sigma$ ). Moreover, if for some $v \in V$, $\mu(\{x \in X \mid x(\mathrm{id})=v\})>0$ then there exists a periodic point $x \in X$ with $x(\mathrm{id})=v$.

Theorem 7.2 If the conjecture above is true, then the surface subgroup conjecture is true. That is, if $\Gamma<\mathrm{PSL}_{2}(\mathbb{C})$ is a cocompact discrete group then there exists a subgroup $\Sigma<\Gamma$ that is isomorphic to the fundamental group of a closed surface of genus at least 2 . 
Proof Let $\Sigma<\mathrm{PSL}_{2}(\mathbb{R})$ be a discrete cocompact surface group. Let $Y$ be any subset of $\Gamma \backslash \mathrm{PSL}_{2}(\mathbb{C})$ that is $\Sigma$-invariant, closed and such that the action of $\Sigma$ on $Y$ is minimal. We claim that there is a $\Sigma$-invariant probability measure supported on $Y$. Consider the set

$$
\tilde{Y}=\left\{(y g, \Sigma g) \in \Gamma \backslash \mathrm{PSL}_{2}(\mathbb{C}) \times \Sigma \backslash \mathrm{PSL}_{2}(\mathbb{R}) \mid y \in Y, g \in \mathrm{PSL}_{2}(\mathbb{R})\right\} .
$$

We claim that this set is closed. To see this, let $\left\{\left(y_{i} g_{i}, \Sigma g_{i}\right)\right\}_{i=1}^{\infty}$ be a sequence in $\tilde{Y}$ where $\left\{y_{i}\right\} \subset Y$ and $\left\{g_{i}\right\} \subset \mathrm{PSL}_{2}(\mathbb{R})$. Let $D \subset \mathrm{PSL}_{2}(\mathbb{R})$ be a compact set such that $\Sigma D=\mathrm{PSL}_{2}(\mathbb{R})$. Then there exists $h_{i} \in \Sigma$ and $d_{i} \in D$ such that $g_{i}=h_{i} d_{i}$. After passing to a subsequence we may assume that $\left\{y_{i} h_{i}\right\} \subset Y$ has a limit point $y_{\infty}$ and that $\left\{d_{i}\right\}$ has a limit point $d_{\infty} \in D$. Thus $\left\{\left(y_{i} g_{i}, \Sigma g_{i}\right)\right\}=\left\{\left(y_{i} h_{i} d_{i}, \Sigma d_{i}\right)\right\}$ has the limit point $\left(y_{\infty} d_{\infty}, \Sigma d_{\infty}\right) \in \widetilde{Y}$. This proves that $\tilde{Y}$ is sequentially compact which implies the claim.

Since $\tilde{Y}$ is invariant under the diagonal action of $\mathrm{PSL}_{2}(\mathbb{R})$ on the right, Ratner's theorems on unipotent flows imply that there exists a $\mathrm{PSL}_{2}(\mathbb{R})$-invariant Borel probability measure $\tilde{\mu}$ supported on $\tilde{Y}$. Now let $K \subset \mathrm{PSL}_{2}(\mathbb{R})$ be a fundamental domain for the action of $\Sigma$. That is, $K$ is a Borel set such that $\Sigma K=\mathrm{PSL}_{2}(\mathbb{R})$ and if $g_{1} \neq g_{2} \in K$ then $g_{1} K \cap g_{2} K$ has Haar measure zero. If $Y_{0} \subset Y$ is Borel then define

$$
\mu\left(Y_{0}\right)=\tilde{\mu}\left(\left\{(y k, \Sigma k) \in \tilde{Y} \mid y \in Y_{0}, k \in K\right\}\right) .
$$

Then $\mu$ is a $\Sigma$-invariant probability measure supported on $Y$. This proves the claim. Let $d$ denote the usual distance function on $\mathrm{PSL}_{2}(\mathbb{C})$. Let $S \subset \Sigma$ be a finite symmetric generating set. Let

$$
\rho=\min \left\{d\left(\mathrm{id}, g \gamma g^{-1}\right): \gamma \in \Gamma-\{\mathrm{id}\}, g \in \mathrm{PSL}_{2}(\mathbb{C})\right\}>0 .
$$

Without loss of generality, let us assume that $\Sigma$ has a presentation of the form $\Sigma=$ $\langle S \mid R\rangle$ where $R$ is a finite set of words in $S$.

Let $\phi: \Gamma \rightarrow \mathrm{PSL}_{2}(\mathbb{C})$ be the inclusion map. Choose $\epsilon_{0}$ so that $0<\epsilon_{0} \leq \epsilon$ and if $r=s_{1} \cdots s_{k}$ is in $R$ (with $s_{i} \in S$ ) if $g_{0}, g_{1}, \ldots, g_{k} \in \mathrm{PSL}_{2}(\mathbb{R})$ are such that $d\left(\phi\left(s_{i}\right), g_{i}\right) \leq \epsilon_{0}$ for all $i$ then $d\left(g_{1} \cdots g_{k}\right.$,id $)<\rho$.

Let $\delta>0$ be such that for all $g_{1}, g_{2} \in \mathrm{PSL}_{2}(\mathbb{C})$ with $d\left(g_{1}\right.$, id $)<\delta_{1}$ and $d\left(g_{2}\right.$,id $)<\delta_{1}$ if $s \in S$ then, $d\left(g_{1} \phi(s) g_{2}, \phi(s)\right)<\epsilon_{0}$.

Let $V=\left\{v_{1}, v_{2}, \ldots, v_{n}\right\}$ be a Borel partition of $Y$ into sets $v_{i}$ of diameter less than $\delta$. Assume that each $v_{i}$ has positive $\mu$-measure.

Let $\mathcal{G}$ be the graph with vertex set $V=\left\{v_{1}, \ldots, v_{n}\right\}$ and edges defined as follows. For each $v, w \in V$, if there exist elements $p \in v, q \in w$ and $s \in S$ such that $p s=q$ then 
there is a directed edge in $\mathcal{G}$ from $v$ to $w$ labeled $s$ which we denote by $(v, w ; s)$. There are no other edges.

Let $X \subset V^{\Sigma}$ be the graph subshift determined by $\mathcal{G}$.

We will choose, for each $x \in X$, an $\epsilon$-perturbation $\phi_{x}$ of $\phi$. To get started, choose a basepoint $p_{i} \in v_{i}$ for each $i$. Assume $p_{1}=\Gamma$.

If there is an edge $e=(v, w ; s)$ in $\mathcal{G}$ then there exists points $p \in v, q \in w$ such that $p s=q$. Let $p_{v}, q_{w}$ be the basepoints of $v$ and $w$ respectively. Because $v$ and $w$ each have diameter at most $\delta$, there exists elements $g_{v}, g_{w} \in \operatorname{PSL}_{2}(\mathbb{C})$ such that $d\left(g_{v}, \mathrm{id}\right)<\delta, d\left(g_{w}, \mathrm{id}\right)<\delta, p_{v} g_{v}=p$ and $q_{w} g_{w}=q$.

Let $\psi_{e}=g_{v} s g_{w}^{-1}$. Note that $p_{v} \psi_{e}=p_{v} g_{v} s g_{w}^{-1}=q_{w}$. By choice of $\delta, d\left(\psi_{e}, s\right)<\epsilon$. There is also an edge $e^{\prime}=\left(w, v: s^{-1}\right)$ in $\mathcal{G}$. We choose $\psi_{e^{\prime}}=\psi_{e}^{-1}$, and observe that $q_{w} \psi_{e^{\prime}}=p_{v}$ and $d\left(\psi_{e^{\prime}}, \phi\left(s^{-1}\right)\right)<\epsilon$.

Let $x \in X$. For $f \in \Sigma$, represent $f$ as $f=t_{1} \cdots t_{m}$ for some $t_{i} \in S$. Let $t_{0}=\mathrm{id}$.

Let $\phi_{x}(f)=\psi_{e_{1}} \cdots \psi_{e_{m}}$ where

$$
e_{i}=\left(x\left(t_{0} \cdots t_{i-1}\right), x\left(t_{1} \cdots t_{i}\right) ; t_{i}\right) .
$$

To show that $\phi_{x}$ is well-defined, it suffices to show that if $r=s_{1} \cdots s_{k} \in R$ is a relator (with $s_{i} \in S$ ) and $e_{1}, e_{2}, \ldots, e_{k}$ is a directed cycle in $\mathcal{G}$ such that $e_{i}$ is labeled $s_{i}$ for all $i$, then $\psi_{e_{1}} \cdots \psi_{e_{k}}=\mathrm{id}$. Let $v$ be the source of $e_{1}$ and $p \in v$ its basepoint. Since $e_{1}, \ldots, e_{k}$ is a directed cycle, $p \psi_{e_{1}} \cdots \psi_{e_{k}}=p$. Thus, if $p=\Gamma g$ for some $g \in \mathrm{PSL}_{2}(\mathbb{C})$, then $\psi_{e_{1}} \cdots \psi_{e_{k}} \in g^{-1} \Gamma g$.

As noted above, $d\left(\psi\left(e_{i}\right), \phi\left(s_{i}\right)\right)<\epsilon_{0}$. So by the choice of $\epsilon_{0}, d\left(\psi_{e_{1}} \cdots \psi_{e_{k}}\right.$, id $)<\rho$. By definition of $\rho$, this implies $\psi_{e_{1}} \cdots \psi_{e_{k}}=\mathrm{id}$. Thus, $\phi_{x}$ is well-defined.

The rest of the proof is exactly the same as the proof of Theorem 1.1 beginning with Lemma 3.1 except in one detail. We must invoke the conjecture above to ensure the existence of a periodic point.

\section{References}

[1] L Bowen, Periodicity and circle packings of the hyperbolic plane, Geom. Dedicata 102 (2003) 213-236 MR2026846

[2] L Bowen, C Holton, C Radin, L Sadun, Uniqueness and symmetry in problems of optimally dense packings, Math. Phys. Electron. J. 11 (2005) Paper 1, 34 pp. MR2122360

[3] M R Bridson, A Haefliger, Metric spaces of non-positive curvature, Grund. der Math. Wissenschaften 319, Springer, Berlin (1999) MR1744486 
[4] M Coornaert, Mesures de Patterson-Sullivan sur le bord d'un espace hyperbolique au sens de Gromov, Pacific J. Math. 159 (1993) 241-270 MR1214072

[5] M Coornaert, T Delzant, A Papadopoulos, Géométrie et théorie des groupes. Les groupes hyperboliques de Gromov, Lecture Notes in Math. 1441, Springer, Berlin (1990) MR1075994 With an English summary

[6] A Dranishnikov, V Schroeder, Aperiodic colorings and tilings of Coxeter groups, Groups Geom. Dyn. 1 (2007) 311-328 MR2314048

[7] V A Efremovič, The proximity geometry of Riemannian manifolds, Uspehi Matem. Nauk (N.S.) 8 (1953) 189-195

[8] C Goodman-Strauss, A strongly aperiodic set of tiles in the hyperbolic plane, Invent. Math. 159 (2005) 119-132 MR2142334

[9] M Gromov, Hyperbolic groups, from: "Essays in group theory", Math. Sci. Res. Inst. Publ. 8, Springer, New York (1987) 75-263 MR919829

[10] M Lackenby, Surface subgroups of Kleinian groups with torsion, to appear in Invent. Math. arXiv:0804.1309

[11] M Lackenby, D D Long, A W Reid, LERF and the Lubotzky-Sarnak conjecture, Geom. Topol. 12 (2008) 2047-2056 MR2431015

[12] G A Margulis, S Mozes, Aperiodic tilings of the hyperbolic plane by convex polygons, Israel J. Math. 107 (1998) 319-325 MR1658579

[13] J D Masters, Kleinian groups with ubiquitous surface subgroups, Groups Geom. Dyn. 2 (2008) 263-269 MR2393182

[14] C T McMullen, Hausdorff dimension and conformal dynamics. I. Strong convergence of Kleinian groups, J. Differential Geom. 51 (1999) 471-515 MR1726737

[15] J Milnor, A note on curvature and fundamental group, J. Differential Geometry 2 (1968) 1-7 MR0232311

[16] R Penrose, Pentaplexity: a class of nonperiodic tilings of the plane, Eureka 39 (1978) 16-32 Reproduced in Math. Intell. 2 (1979) 32-37 MR558670

[17] A S Švarc, A volume invariant of coverings, Dokl. Akad. Nauk SSSR (N.S.) 105 (1955) 32-34 MR0075634

[18] W A Veech, Teichmüller curves in moduli space, Eisenstein series and an application to triangular billiards, Invent. Math. 97 (1989) 553-583 MR1005006

Department of Mathematics, University of Hawaii

Honolulu, HI 96822, USA

lpbowen@math.hawaii.edu

Proposed: Martin Bridson

Received: 27 May 2007

Seconded: Benson Farb, Jean-Pierre Otal

Revised: 26 August 2009 